\title{
Synthesis of Allenamides by Copper-Catalyzed Coupling of Propargylic Bromides and Nitrogen Nucleophiles
}

\author{
Charles S. Demmer, Emeline Benoit and Gwilherm Evano* \\ Laboratoire de Chimie Organique, Service de Chimie et PhysicoChimie Organiques, Université \\ Libre de Bruxelles, Avenue F. D. Roosevelt 50, CP160/06, 1050 Brussels, Belgium.
}

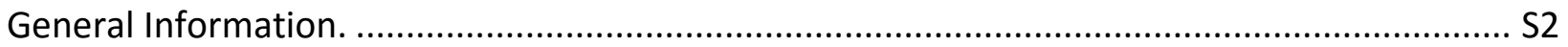

Experimental Procedures and Characterization Data:

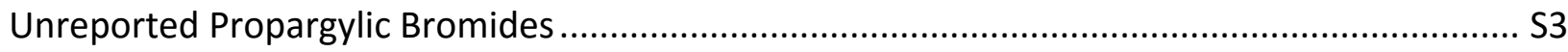

Experimental Procedures and Characterization Data:

Synthesis of Allenamides from Propargylic Bromides ......................................................... S6

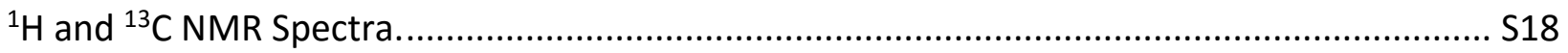




\section{General Information.}

All reactions were carried out in oven-dried glassware under an argon atmosphere employing standard techniques in handling air-sensitive materials. All solvents were reagent grade.

Copper(I) thiophene carboxylate (CUTC) was synthesized according to the literature $.{ }^{51} 2,2^{\prime}-$ bipyridine, 4,4'-dimethyl-2,2'-bipyridine were respectively purchased from Aldrich and $\mathrm{TCl}$ and used as supplied. Finely powdered anhydrous cesium carbonate and potassium phosphonate were respectively purchased from Acros and Aldrich and used as supplied. All other reagents were used as supplied.

Reactions were magnetically stirred and monitored by thin layer chromatography using Merck-Kiesegel $60 \mathrm{~F}_{254}$ plates. Flash chromatography was performed with Geduran ${ }^{\circledR}$ Si 60 (particle size 40-63 $\mu \mathrm{m}$ ) supplied by Merck. Yields refer to chromatographically and spectroscopically pure compounds.

Proton NMR spectra were recorded using an internal deuterium lock at ambient temperature on a Bruker $300 \mathrm{MHz}$ spectrometers. Internal reference of $\delta_{H} 7.26$ was used for $\mathrm{CDCl}_{3}$. Data are presented as follows: chemical shift (in ppm on the $\delta$ scale relative to $\delta_{\text {TMS }}=0$ ), multiplicity $(\mathrm{s}=$ singlet, $\mathrm{d}=$ doublet, $\mathrm{t}=$ triplet, $\mathrm{q}=$ quartet, quint. = quintuplet, sext. = sextuplet, sept. = septuplet, $\mathrm{m}=$ multiplet, br. = broad, app. = apparent $)$, coupling constant $(\mathrm{J} / \mathrm{Hz})$ and integration. Resonances that are either partially or fully obscured are denoted obscured (obs.). Carbon-13 NMR spectra were recorded at $75 \mathrm{MHz}$ using $\mathrm{CDCl}_{3}\left(\delta_{c} 77.16\right)$ as internal reference.

Melting points were recorded on a Stuart Scientific Analogue SMP11. Infrared spectra were recorded on a Bruker Alpha (ATR). High-resolution mass-spectra were obtained on Waters Qtof Micro, Waters QTof API US or Thermo Finnigan MAT 95XP spectrometers.

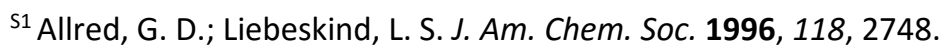




\section{Experimental Procedures and Characterization Data: Unreported Propargylic Bromides}

\section{General procedure:}

To an oven-dried round bottom flask containing the corresponding propargylic alcohol (1.0 equiv.) was added dropwise phosphorus tribromide (0.4 equiv.) at $-20{ }^{\circ} \mathrm{C}$ (or at $-10{ }^{\circ} \mathrm{C}$ if the starting alcohol is a solid). The reaction was slowly warmed up to room temperature and stirred for $1 \mathrm{~h}$. Distillation of the crude reaction mixture under reduced pressure at $50^{\circ} \mathrm{C}$ yields the desired propargylic bromide as a clear and colorless oil.

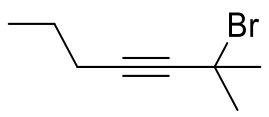

2-Bromo-2-methylhept-3-yne. Reaction run on a $21.7 \mathrm{mmol}$ scale. Yield: $48 \%$ (2.0 g, $10.4 \mathrm{mmol})$. ${ }^{1} \mathrm{H}$ NMR $\left(300 \mathrm{MHz}, \mathrm{CDCl}_{3}\right) \delta 2.20(\mathrm{t}, J=7.0 \mathrm{~Hz}, 2 \mathrm{H}), 2.00(\mathrm{~s}, 6 \mathrm{H}), 1.61-1.46(\mathrm{~m}, 2 \mathrm{H}), 0.98(\mathrm{t}, J=$ $7.4 \mathrm{~Hz}, 3 \mathrm{H}) ;{ }^{13} \mathrm{C}$ NMR $\left(75 \mathrm{MHz}, \mathrm{CDCl}_{3}\right): \delta 85.8,84.9,50.0,36.9,21.9,20.8,13.4$; IR (ATR): $v_{\max }$ 2992, 2964, 2933, 2872, 2236, 1458, 1381, 1365, 1256, 1111, 1079, 1066, 1012, 904, $673 \mathrm{~cm}^{-1}$; EIHRMS m/z calcd for $\mathrm{C}_{8} \mathrm{H}_{13} \mathrm{Br}[\mathrm{M}]^{+}$188.0195, found 188.0197 .

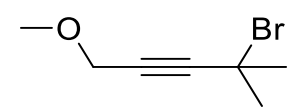

4-Bromo-1-methoxy-4-methylpent-2-yne. Reaction run on a $18.7 \mathrm{mmol}$ scale. Yield: $67 \%$ (2.4 g, $12.5 \mathrm{mmol}) .{ }^{1} \mathrm{H} \mathrm{NMR}\left(300 \mathrm{MHz}, \mathrm{CDCl}_{3}\right): \delta 4.14(\mathrm{~s}, 2 \mathrm{H}), 3.38(\mathrm{~s}, 3 \mathrm{H}), 2.02(\mathrm{~s}, 6 \mathrm{H}) ;{ }^{13} \mathrm{C} \mathrm{NMR}(75 \mathrm{MHz}$, $\left.\mathrm{CDCl}_{3}\right): \delta 90.5,80.6,60.0,57.7,48.1,36.4$; IR (ATR): $v_{\max } 2993,2975,2929,2821,2362,2340$, 1449, 1366, 1356, 1253, 1187, 1106, 1057, 910, $891 \mathrm{~cm}^{-1}$; ElHRMS m/z calcd for $\mathrm{C}_{7} \mathrm{H}_{11} \mathrm{BrO}[\mathrm{M}]^{+}$ 189.9988, found 189.9994. 


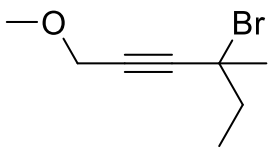

4-Bromo-1-methoxy-4-methylhex-2-yne. Reaction run on a $13.3 \mathrm{mmol}$ scale. Yield: 62\% (1.5 g, $8.2 \mathrm{mmol}) .{ }^{1} \mathrm{H}$ NMR $\left(300 \mathrm{MHz}, \mathrm{CDCl}_{3}\right): \delta 4.12(\mathrm{~s}, 2 \mathrm{H}), 3.34(\mathrm{~s}, 3 \mathrm{H}), 2.07-1.87(\mathrm{~m}, 5 \mathrm{H}), 1.12(\mathrm{t}, J=$ $7.2 \mathrm{~Hz}, 3 \mathrm{H}) ;{ }^{13} \mathrm{C}$ NMR $\left(75 \mathrm{MHz}, \mathrm{CDCl}_{3}\right): \delta 88.9,82.0,59.9,57.5,55.4,40.3,34.1,11.3$; IR (ATR): $v_{\max }$ 2974, 2936, 2821, 2337, 1450, 1376, 1356, 1217, 1187, 1103, 1034, 1001, 907, $807 \mathrm{~cm}^{-1}$; EIHRMS $\mathrm{m} / \mathrm{z}$ calcd for $\mathrm{C}_{8} \mathrm{H}_{13} \mathrm{BrO}[\mathrm{M}]^{+}$204.0144, found 204.0151.

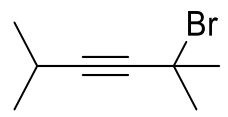

2-Bromo-2,5-dimethylhex-3-yne. Reaction run on a $11.9 \mathrm{mmol}$ scale. Yield: 47\% (1.1 g, 5.6 mmol). ${ }^{1} \mathrm{H}$ NMR (300 MHz, $\mathrm{CDCl}_{3}$ ) $\delta 2.58$ (sept., $J=6.9 \mathrm{~Hz}, 1 \mathrm{H}$ ), $1.98(\mathrm{~s}, 6 \mathrm{H}), 1.14$ (d, $J=6.9 \mathrm{~Hz}$, $6 \mathrm{H}) ;{ }^{13} \mathrm{C} \mathrm{NMR}\left(75 \mathrm{MHz}, \mathrm{CDCl}_{3}\right): \delta 91.3,84.1,50.4,37.0,22.8,20.7$. IR (ATR): $v_{\max } 2971,2929,2873$, 2230, 1458, 1365, 1320, 1255, 1164, 1113, 1102, 1011, 928, 834, $664 \mathrm{~cm}^{-1}$; ElHRMS m/z calcd for $\mathrm{C}_{8} \mathrm{H}_{13} \mathrm{Br}[\mathrm{M}]^{+}$188.0195, found 188.0198.

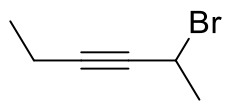

2-Bromohex-3-yne. Reaction run on a $6.1 \mathrm{mmol}$ scale. Yield: $41 \%$ (400 mg, $2.5 \mathrm{mmol}) .{ }^{1} \mathrm{H}$ NMR $\left(300 \mathrm{MHz}, \mathrm{CDCl}_{3}\right): \delta 4.65$ (qt, $J=6.8$ and $\left.2.1 \mathrm{~Hz}, 1 \mathrm{H}\right), 2.29$ (qd, $J=7.5$ and $2.0 \mathrm{~Hz}, 2 \mathrm{H}$ ), $1.93(\mathrm{~d}, J=$ $6.8 \mathrm{~Hz}, 3 \mathrm{H}), 1.18(\mathrm{t}, \mathrm{J}=7.5 \mathrm{~Hz}, 3 \mathrm{H}) ;{ }^{13} \mathrm{C} \mathrm{NMR}\left(75 \mathrm{MHz} \mathrm{CDCl}_{3}\right): \delta 88.9,80.0,32.8,28.0,13.6,12.7$; IR (ATR): $v_{\max } 2977,2937,2925,2878,2234,1441,1374,1321,1187,1066,1034,975,922,704$ $\mathrm{cm}^{-1}$.

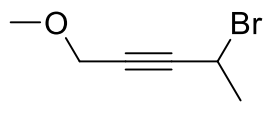

4-Bromo-1-methoxypent-2-yne. Reaction run on a $13.3 \mathrm{mmol}$ scale. Yield: $62 \%$ (1.5 g, $8.2 \mathrm{mmol})$. ${ }^{1} \mathrm{H} \operatorname{NMR}\left(300 \mathrm{MHz}, \mathrm{CDCl}_{3}\right): \delta 4.75-4.63(\mathrm{~m}, 1 \mathrm{H}), 4.15(\mathrm{~d}, J=1.7 \mathrm{~Hz}, 2 \mathrm{H}), 3.42(\mathrm{~s}, 3 \mathrm{H}), 1.96(\mathrm{~d}, J=$ $6.9 \mathrm{~Hz}, 3 \mathrm{H}) ;{ }^{13} \mathrm{C} \mathrm{NMR}\left(75 \mathrm{MHz}, \mathrm{CDCl}_{3}\right): \delta 86.6,82.1,60.0,57.8,30.8,27.4$; IR (ATR): $v_{\max } 2992$, 
2929, 2822, 2362, 2336, 1445, 1375, 1312, 1185, 1099, 952, $904 \mathrm{~cm}^{-1}$; ElHRMS m/z calcd for $\mathrm{C}_{6} \mathrm{H}_{9} \mathrm{BrO}[\mathrm{M}]^{+}$175.9831, found 175.9834 .

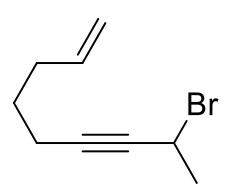

8-Bromonon-1-en-6-yne. Reaction run on a $11.2 \mathrm{mmol}$ scale. Yield: 26\% (600 mg, $3.0 \mathrm{mmol}, 90 \%$ purity). ${ }^{1} \mathrm{H}$ NMR $\left(300 \mathrm{MHz}, \mathrm{CDCl}_{3}\right): \delta 5.91-5.75(\mathrm{~m}, 1 \mathrm{H}), 5.14-4.98(\mathrm{~m}, 2 \mathrm{H}), 4.69$ (app. qt, $J=6.8$ and $2.1 \mathrm{~Hz}, 1 \mathrm{H}$ ), $2.30(\mathrm{td}, J=7.1$ and $2.2 \mathrm{~Hz}, 2 \mathrm{H}), 2.24-2.14(\mathrm{~m}, 2 \mathrm{H}), 1.94(\mathrm{~d}, J=6.9 \mathrm{~Hz}, 3 \mathrm{H}), 1.65$ (app. quint., $J=7.2 \mathrm{~Hz}, 2 \mathrm{H}$ ); ${ }^{13} \mathrm{C} \mathrm{NMR}\left(75 \mathrm{MHz}, \mathrm{CDCl}_{3}\right): \delta 137.8,115.4,87.2,81.0,32.8,32.7,28.0$, 27.6, 18.4; IR (ATR): $v_{\max } 3076,2995,2977,2929,2862,2842,2240,1640,1440,1374,1315,1186$, 1067, 992, 956, $913 \mathrm{~cm}^{-1}$; ElHRMS m/z calcd for $\mathrm{C}_{9} \mathrm{H}_{13} \mathrm{Br}[\mathrm{M}]^{+} 200.0195$, found 200.0189 . 


\section{Experimental Procedure and Characterization Data: Synthesis of Allenamides from Propargylic Bromides}

\section{General procedure:}

A $15 \mathrm{~mL}$ pressure tube was charged with copper(I) thiophene carboxylate (22.9 mg, $20 \mathrm{~mol} \%)$, 2,2'-bipyridine (37.5 mg, $40 \mathrm{~mol} \%$ ) or 4,4'-dimethyl-2,2'-bipyridine (44.2 $\mathrm{mg}, 40 \mathrm{~mol} \%$ ) and cesium carbonate $(293 \mathrm{mg}, 0.90 \mathrm{mmol})$ or potassium phosphate $(191 \mathrm{mg}, 0.90 \mathrm{mmol})$; if solid, the nitrogen nucleophile $(0.60 \mathrm{mmol})$ was also introduced at this stage. The tube was fitted with a rubber septum, evacuated under high vacuum and backfilled with argon. Dry and degassed acetonitrile $(1 \mathrm{~mL})$ was next added as well as the nitrogen nucleophile $(0.60 \mathrm{mmol})$ which was added at this stage if liquid, followed by the propargylic bromide $(1.2 \mathrm{mmol})$. The rubber septum was replaced by Teflon-coated screw cap and the heterogeneous suspension was stirred overnight at room temperature unless otherwise stated. The crude mixture was filtered through a plug of Celite ${ }^{\circledR}$ (washed with EtOAc). The filtrate was concentrated and the residue was dissolved in EtOAc $(10 \mathrm{~mL})$ before being successively washed with a saturated aqueous solution of copper(II)sulfate $(10 \mathrm{~mL})$ and brine $(10 \mathrm{~mL})$, dried over magnesium sulfate, filtered and concentrated under reduced pressure. The crude residue was purified by flash column chromatography over triethylamine-deactivated silica gel.

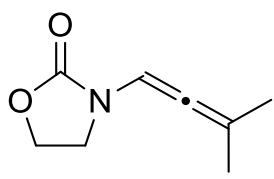

$1 \mathrm{a}$

3-(3-Methylbuta-1,2-dien-1-yl)oxazolidin-2-one 1a. ${ }^{\mathrm{S} 2}$ Reaction run on a $1.0 \mathrm{mmol}$ scale. Yield: $52 \%$ ( $82.0 \mathrm{mg}, 0.52 \mathrm{mmol}$ ). Solvent system for flash column chromatography: EtOAc/petroleum ether/Et 3 N: 20/75/5; Yellow oil; ${ }^{1} \mathrm{H}$ NMR $\left(300 \mathrm{MHz}, \mathrm{CDCl}_{3}\right): \delta 6.65-6.50$ (br. m, 1H), 4.37 (t, $J=$ $7.9 \mathrm{~Hz}, 2 \mathrm{H}), 3.53(\mathrm{t}, J=7.9 \mathrm{~Hz}, 2 \mathrm{H}), 1.77(\mathrm{~s}, 6 \mathrm{H}) ;{ }^{13} \mathrm{C} \mathrm{NMR}\left(75 \mathrm{MHz}, \mathrm{CDCl}_{3}\right): \delta$ 191.4, 155.6, 108.9,

\footnotetext{
s2 This compound has been previously reported: Trost, B. M.; Stiles, D. T. Org. Lett. 2005, 7, 2117.
} 
94.2, 62.2, 43.4, 22.3; IR (ATR): $v_{\max } 2982,2912,1964,1758,1485,1433,1392,1249,1081,1035$, 753, $665 \mathrm{~cm}^{-1}$; ESIHRMS $\mathrm{m} / \mathrm{z}$ calcd for $\mathrm{C}_{8} \mathrm{H}_{12} \mathrm{NO}_{2}[\mathrm{M}+\mathrm{H}]^{+}$154.0863, found 154.0865 .

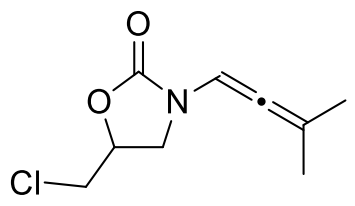

$1 \mathrm{~b}$

5-(Chloromethyl)-3-(3-methylbuta-1,2-dien-1-yl)oxazolidin-2-one 1b. Reaction run on a 1.0 mmol scale. Yield: $71 \%(143.0 \mathrm{mg}, 0.71 \mathrm{mmol}$ ). Solvent system for flash column chromatography: EtOAc/petroleum ether/Et ${ }_{3} \mathrm{~N}$ : 20/75/5; Yellow oil; ${ }^{1} \mathrm{H}$ NMR (300 MHz, $\left.\mathrm{CDCl}_{3}\right): \delta 6.63-6.55$ (br. $\mathrm{m}, 1 \mathrm{H}), 4.83-4.70(\mathrm{~m}, 1 \mathrm{H}), 3.77-3.60(\mathrm{~m}, 3 \mathrm{H}), 3.53-3.32(\mathrm{~m}, 1 \mathrm{H}), 1.80(\mathrm{~d}, J=2.8 \mathrm{~Hz}, 6 \mathrm{H}) ;{ }^{13} \mathrm{C}$ $\operatorname{NMR}\left(75 \mathrm{MHz}, \mathrm{CDCl}_{3}\right): \delta$ 191.4, 154.3, 109.4, 93.9, 72.0, 46.7, 44.7, 22.4; IR (ATR): $v_{\max } 2984$, 2940, 2912, 2854, 1966, 1750, 1485, 1435, 1388, 1362, 1249, 1090, 1049, 749, $672 \mathrm{~cm}^{-1}$; ESIHRMS $m / z$ calcd for $\mathrm{C}_{9} \mathrm{H}_{13} \mathrm{NO}_{2} \mathrm{Cl}[\mathrm{M}+\mathrm{H}]^{+} 202.0629$, found 202.0633 .

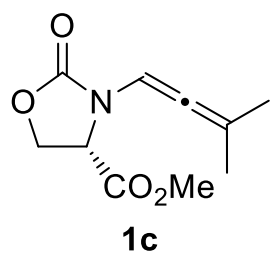

Methyl (S)-3-(3-methylbuta-1,2-dien-1-yl)oxazolidin-2-one-4-carboxylate 1c. Reaction run on a $1.0 \mathrm{mmol}$ scale. Yield: $67 \%(142.0 \mathrm{mg}, 0.67 \mathrm{mmol})$. Solvent system for flash column chromatography: EtOAc/petroleum ether/Et ${ }_{3} \mathrm{~N}$ : 10/87.5/2.5; Yellow solid; Mp: $59{ }^{\circ} \mathrm{C}$; ${ }^{1} \mathrm{H}$ NMR $\left(300 \mathrm{MHz}, \mathrm{CDCl}_{3}\right.$ ): $\delta 6.68-6.52$ (br. $\mathrm{m}, 1 \mathrm{H}$ ), 4.53 ( $\mathrm{A}$ of $\mathrm{ABX}$ syst., $J=8.4$ and $8.4 \mathrm{~Hz}, 1 \mathrm{H}$ ), $4.39-$ $4.23(\mathrm{~m}, 2 \mathrm{H}), 3.77(\mathrm{~s}, 3 \mathrm{H}), 1.77(\mathrm{~d}, J=2.2 \mathrm{~Hz}, 3 \mathrm{H}), 1.69(\mathrm{~d}, J=2.1 \mathrm{~Hz}, 3 \mathrm{H}) ;{ }^{13} \mathrm{C} \mathrm{NMR}\left(75 \mathrm{MHz}, \mathrm{CDCl}_{3}\right)$ : $\delta 191.1,169.9,154.8,110.5,93.6,64.9,56.8,52.9,22.1$; IR (ATR): $v_{\max } 2975,2949,2918,1968$, 1750, 1707, 1478, 1430, 1399, 1367, 1314, 1225, 1090, 1066, 1043, 989, $755 \mathrm{~cm}^{-1}$; ESIHRMS m/z calcd for $\mathrm{C}_{10} \mathrm{H}_{14} \mathrm{NO}_{4}[\mathrm{M}+\mathrm{H}]^{+} 212.0917$, found 212.0919. 


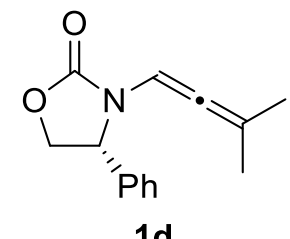

(R)-3-(3-Methylbuta-1,2-dien-1-yl)-4-phenyloxazolidin-2-one 1d. ${ }^{\mathrm{S3}}$ Yield: 44\% (100.0 mg, 0.26 $\mathrm{mmol}$ ). Solvent system for flash column chromatography: EtOAc/petroleum ether/Et ${ }_{3} \mathrm{~N}: 10 / 85 / 5$; Yellow solid; $\mathrm{Mp}: 78{ }^{\circ} \mathrm{C} ;{ }^{1} \mathrm{H}$ NMR $\left(300 \mathrm{MHz}, \mathrm{CDCl}_{3}\right): \delta 7.43-7.17(\mathrm{~m}, 5 \mathrm{H}), 6.62-6.46$ (br. m, $\left.1 \mathrm{H}\right)$, 4.81 (X of $A B X$ syst., $J=8.9$ and $6.2 \mathrm{~Hz}, 1 \mathrm{H}$ ), 4.68 ( $\mathrm{A}$ of $A B X$ syst., $J=8.8 \mathrm{~Hz}$ and $8.8 \mathrm{~Hz}, 1 \mathrm{H}$ ), 4.12 (B of $A B X$ syst., $J=8.5$ and $6.2 \mathrm{~Hz}, 1 \mathrm{H}), 1.64(\mathrm{~d}, J=2.3 \mathrm{~Hz}, 3 \mathrm{H}), 1.00(\mathrm{~d}, J=2.3 \mathrm{~Hz}, 3 \mathrm{H}) ;{ }^{13} \mathrm{C} \mathrm{NMR}$ (75 $\mathrm{MHz} \mathrm{CDCl}_{3}$ ): $\delta$ 192.9, 155.9, 139.1, 129.1, 128.5, 126.5, 109.0, 93.0, 70.5, 59.6, 22.0, 21.0; IR (FTIR): $v_{\max } 2978,2919,2859,1972,1738,1476,1458,1428,1395,1363,1327,1294,1240$, $1219,1088,1079,1054,1032,804,762,750,698 \mathrm{~cm}^{-1}$; ESIHRMS m/z calcd for $\mathrm{C}_{14} \mathrm{H}_{16} \mathrm{NO}_{2}[\mathrm{M}+\mathrm{H}]^{+}$ 230.1176, found 230.1179 .

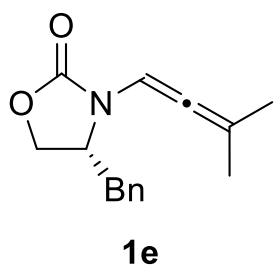

(R)-4-Benzyl-3-(3-methylbuta-1,2-dien-1-yl)oxazolidin-2-one 1e. ${ }^{\mathrm{S3}}$ Yield: 43\% (106.0 mg, 0.26 $\mathrm{mmol}$ ). Solvent system for flash column chromatography: EtOAc/petroleum ether/Et ${ }_{3} \mathrm{~N}: 10 / 85 / 5$; Amorphous yellow solid; ${ }^{1} \mathrm{H}$ NMR $\left(300 \mathrm{MHz} \mathrm{CDCl}_{3}\right): \delta 7.40-7.19(\mathrm{~m}, 3 \mathrm{H}), 7.15-7.05(\mathrm{~m}, 2 \mathrm{H})$, $6.69-6.60$ (br. m, 1H), $4.23-4.13$ (A of ABX syst., $J=8.6$ and $8.6 \mathrm{~Hz}, 1 \mathrm{H}$ ), 4.09 (B of ABX syst., $J$ $=8.8$ and $4.0 \mathrm{~Hz}, 1 \mathrm{H}), 4.01(\mathrm{~m}, 1 \mathrm{H}), 3.16\left(\mathrm{~A}^{\prime}\right.$ of $\mathrm{A}^{\prime} \mathrm{B}^{\prime} \mathrm{X}$ syst., $J=13.6$ and $\left.2.8 \mathrm{~Hz}, 1 \mathrm{H}\right), 2.68\left(\mathrm{~B}^{\prime}\right.$ of $\mathrm{A}^{\prime} \mathrm{B}^{\prime} X$ syst., $J=13.6$ and $\left.9.1 \mathrm{~Hz}, 1 \mathrm{H}\right), 1.92-1.83$ (app. $\left.\mathrm{t}, J=3.0 \mathrm{~Hz}, 6 \mathrm{H}\right) ;{ }^{13} \mathrm{C} \mathrm{NMR}\left(75 \mathrm{MHz}, \mathrm{CDCl}_{3}\right)$ : $\delta 192.0,155.3,135.8,129.3,129.1,127.3,108.7,93.3,66.6,56.0,36.9,22.3,22.1 ;$ IR (FTIR): $v_{\max }$

\footnotetext{
\$3 This compound has been previously reported: Shen, L.; Hsung, R. P.; Zhang, Y.; Antoline, J. E.; Zhang, X. Org. Lett. 2005, 7, 3081.
} 
$3028,2980,2936,2915,1966,1749,1480,1431,1397,1238,1087,1008,911,734,702,678$ $\mathrm{cm}^{-1}$; ESIHRMS $\mathrm{m} / \mathrm{z}$ calcd for $\mathrm{C}_{15} \mathrm{H}_{18} \mathrm{NO}_{2}[\mathrm{M}+\mathrm{H}]^{+} 244.1332$, found 244.1338.

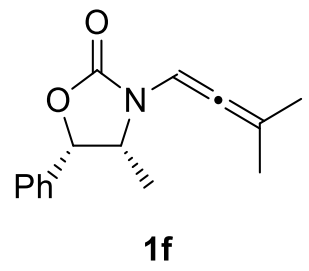

(4R,5S)-4-Methyl-3-(3-methylbuta-1,2-dien-1-yl)-5-phenyloxazolidin-2-one 1f. ${ }^{\mathrm{S} 2}$ Reaction run on a $1.0 \mathrm{mmol}$ scale. Yield: $73 \%(177.6 \mathrm{mg}, 0.73 \mathrm{mmol})$. Solvent system for flash column chromatography: EtOAc/petroleum ether/Et ${ }_{3} \mathrm{~N}$ : 10/85/5; Yellow solid; Mp: $72{ }^{\circ} \mathrm{C}$; ${ }^{1} \mathrm{H}$ NMR $(300$ $\mathrm{MHz}, \mathrm{CDCl}_{3}$ ): $\delta 7.64-7.22(\mathrm{~m}, 5 \mathrm{H}), 6.85-6.43$ (br. $\left.\mathrm{m}, 1 \mathrm{H}\right), 5.67$ (d, J= $7.8 \mathrm{~Hz}, 1 \mathrm{H}$ ), 4.13 (app. quint., $J=6.8 \mathrm{~Hz}, 1 \mathrm{H}$ ), 1.79 (app. dd, $J=7.4$ and $2.3 \mathrm{~Hz}, 6 \mathrm{H}$ ), 0.79 (d, $J=6.5 \mathrm{~Hz}, 3 \mathrm{H}) ;{ }^{13} \mathrm{C} \mathrm{NMR}(75$ $\mathrm{MHz}_{\mathrm{CDCl}}$ ): $\delta 191.9,154.7,134.7,128.7,128.6,126.1,108.4,93.1,79.1,55.1,22.2,21.6,13.9$; IR (ATR): $v_{\max } 2980,2940,2913,1977,1733,1479,1433,1378,1354,1237,1087,974,759,718$, $697,677 \mathrm{~cm}^{-1}$; ESIHRMS $\mathrm{m} / z$ calcd for $\mathrm{C}_{15} \mathrm{H}_{18} \mathrm{NO}_{2}[\mathrm{M}+\mathrm{H}]^{+} 244.1332$, found 244.1334.

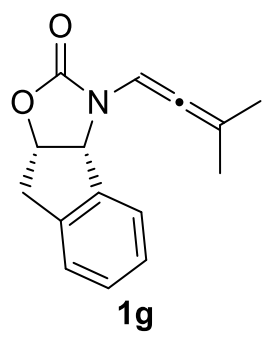

(3aR,8aS)-3-(3-Methylbuta-1,2-dien-1-yl)-3,3a,8,8a-tetrahydro-2H-indeno[1,2-d]oxazol-2-one 1g. Reaction run on a $0.3 \mathrm{mmol}$ scale. Yield: $47 \%(36.0 \mathrm{mg}, 0.15 \mathrm{mmol})$. Solvent system for flash column chromatography: EtOAc/petroleum ether/Et $3 \mathrm{~N}$ : 10/85/5; Yellow solid; Mp: $77^{\circ} \mathrm{C} ;{ }^{1} \mathrm{H}$ NMR (300 MHz, $\left.\mathrm{CDCl}_{3}\right): \delta 7.51-7.41(\mathrm{~m}, 1 \mathrm{H}), 7.38-7.20(\mathrm{~m}, 3 \mathrm{H}), 6.67$ (br. $\left.\mathrm{m}, 1 \mathrm{H}\right), 5.35-5.25(\mathrm{~m}, 1 \mathrm{H})$, $5.22(\mathrm{~d}, J=6.9 \mathrm{~Hz}, 1 \mathrm{H}), 3.37(\mathrm{~s}, 2 \mathrm{H}), 1.99(\mathrm{~s}, 3 \mathrm{H}), 1.89(\mathrm{~s}, 3 \mathrm{H}) ;{ }^{13} \mathrm{C} \mathrm{NMR}\left(75 \mathrm{MHz}, \mathrm{CDCl}_{3}\right): \delta 191.6$, 154.9, 140.3, 139.1, 129.6, 127.5, 126.3, 125.6, 109.1, 94.1, 78.4, 63.8, 38.5, 22.6, 21.9; IR (FTIR): 
$V_{\max } 2957,2916,2849,1959,1750,1475,1431,1372,1329,1227,1193,1104,1033,752,732$, $672 \mathrm{~cm}^{-1}$; ESIHRMS $\mathrm{m} / z$ calcd for $\mathrm{C}_{15} \mathrm{H}_{16} \mathrm{NO}_{2}[\mathrm{M}+\mathrm{H}]^{+} 242.1176$, found 242.1180 .

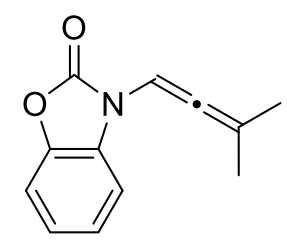

$1 \mathrm{~h}$

3-(3-Methylbuta-1,2-dien-1-yl)benzo[d]oxazol-2(3H)-one 1 h. Reaction run on a $1.0 \mathrm{mmol}$ scale. Yield: $60 \%(120.0 \mathrm{mg}, 0.60 \mathrm{mmol})$. Solvent system for flash column chromatography: EtOAc/petroleum ether/Et $3 \mathrm{~N}$ : 10/85/5; Yellow solid; Mp: $55^{\circ} \mathrm{C} ;{ }^{1} \mathrm{H}$ NMR $\left(300 \mathrm{MHz}, \mathrm{CDCl}_{3}\right): \delta 7.32$ - $7.10(\mathrm{~m}, 4 \mathrm{H}), 6.92-6.84$ (br. $\mathrm{m}, 1 \mathrm{H}), 1.98$ (d, $J=2.4 \mathrm{~Hz}, 6 \mathrm{H}) ;{ }^{13} \mathrm{C} \mathrm{NMR}\left(75 \mathrm{MHz}, \mathrm{CDCl}_{3}\right): \delta 193.4$, 152.6, 142.6, 129.7, 124.1, 123.0, 110.2, 109.9, 109.7, 90.6, 22.0; IR (ATR): $v_{\max } 2980,2940,2910$, $1971,1774,1481,1459,1393,1257,1020,984,742,687 \mathrm{~cm}^{-1}$; ESIHRMS m/z calcd for $\mathrm{C}_{12} \mathrm{H}_{12} \mathrm{NO}_{2}$ $[\mathrm{M}+\mathrm{H}]^{+}$202.0863, found 202.0865 .

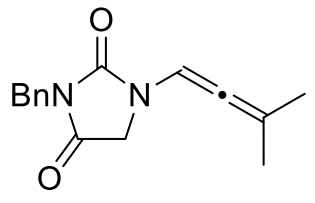

$1 \mathrm{i}$

3-Benzyl-1-(3-methylbuta-1,2-dien-1-yl)imidazolidine-2,4-dione 1i. Reaction run on a $1.0 \mathrm{mmol}$ scale with 4 equiv. of propargylic bromide. Yield: $83 \%$ (213.0 mg, $0.83 \mathrm{mmol}$ ); Solvent system for flash column chromatography: EtOAc/petroleum ether/Et ${ }_{3} \mathrm{~N}$ : 10/85/5; Yellow oil; ${ }^{1} \mathrm{H}$ NMR (300 $\mathrm{MHz}_{\mathrm{CDCl}}$ ): $\delta 7.47-7.38(\mathrm{~m}, 2 \mathrm{H}), 7.38-7.27(\mathrm{~m}, 3 \mathrm{H}), 6.87-6.69$ (br. m, $\left.1 \mathrm{H}\right), 4.67(\mathrm{~s}, 2 \mathrm{H}), 3.83$ (s, 2H), $1.79(\mathrm{~d}, J=2.4 \mathrm{~Hz}, 6 \mathrm{H}) ;{ }^{13} \mathrm{C} \mathrm{NMR}\left(75 \mathrm{MHz}, \mathrm{CDCl}_{3}\right): \delta 191.1,169.0,154.0,135.9,128.9$, 128.8, 128.1, 110.2, 92.6, 48.7, 42.7, 22.3; IR (ATR): $v_{\max } 2983,2940,2910,1966,1773,1712$, 1477, 1448, 1413, 1352, 1301, 1224, 1139, 747, 734, 699, $680 \mathrm{~cm}^{-1}$; ESIHRMS m/z calcd for $\mathrm{C}_{15} \mathrm{H}_{17} \mathrm{~N}_{2} \mathrm{O}_{2}[\mathrm{M}+\mathrm{H}]^{+}$257.1285, found 257.1287. 


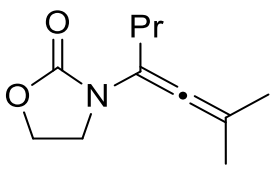

$1 \mathrm{~m}$

3-(2-Methylhepta-2,3-dien-4-yl)oxazolidin-2-one $1 \mathrm{~m}$. Yield: $41 \%$ (48.0 mg, $0.25 \mathrm{mmol}$ ). Solvent system for flash column chromatography: EtOAc/petroleum ether/Et $3 \mathrm{~N}: 15 / 80 / 5$; Colorless oil; ${ }^{1} \mathrm{H} \mathrm{NMR}\left(300 \mathrm{MHz}, \mathrm{CDCl}_{3}\right): \delta 4.29(\mathrm{t}, J=8.0 \mathrm{~Hz}, 2 \mathrm{H}), 3.59(\mathrm{t}, J=8.0 \mathrm{~Hz}, 2 \mathrm{H}), 2.50(\mathrm{t}, J=7.3 \mathrm{~Hz}, 2 \mathrm{H})$, $1.74(\mathrm{~s}, 6 \mathrm{H}), 1.42$ (app. sext., $J=7.4 \mathrm{~Hz}, 2 \mathrm{H}), 0.91$ (t, $J=7.4 \mathrm{~Hz}, 3 \mathrm{H}$ ); ${ }^{13} \mathrm{C} \mathrm{NMR}\left(75 \mathrm{MHz}, \mathrm{CDCl}_{3}\right): \delta$ 193.5, 155.7, 108.4, 105.5, 61.6, 46.6, 31.6, 21.9, 20.6, 13.6; IR (ATR): $v_{\max } 2967,2934,2876,1750$, 1683, 1405, 1228 1081, 1039, 987, 968, 920, 759, $706 \mathrm{~cm}^{-1}$; ESIHRMS $\mathrm{m} / z$ calcd for $\mathrm{C}_{11} \mathrm{H}_{18} \mathrm{NO}_{2}$ $[\mathrm{M}+\mathrm{H}]^{+} 196.1332$, found 196.1328 .

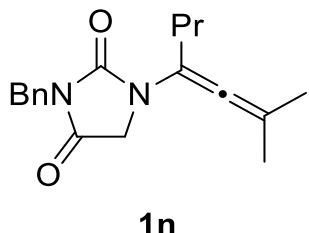

3-Benzyl-1-(2-methylhepta-2,3-dien-4-yl)imidazolidine-2,4-dione 1n. Yield: 78\% (140.0 mg, 0.47 $\mathrm{mmol}$ ). Solvent system for flash column chromatography: EtOAc/petroleum ether/Et ${ }_{3} \mathrm{~N}: 5 / 90 / 5$; Colorless oil; ${ }^{1} \mathrm{H}$ NMR $\left(300 \mathrm{MHz}, \mathrm{CDCl}_{3}\right): \delta 7.45-7.38(\mathrm{~m}, 2 \mathrm{H}), 7.38-7.16(\mathrm{~m}, 3 \mathrm{H}), 4.63(\mathrm{~s}, 2 \mathrm{H})$, $3.86(\mathrm{~s}, 2 \mathrm{H}), 2.54(\mathrm{t}, J=7.3 \mathrm{~Hz}, 2 \mathrm{H}), 1.76(\mathrm{~s}, 6 \mathrm{H}), 1.43$ (app. sext., $J=7.3 \mathrm{~Hz}, 2 \mathrm{H}), 0.93(\mathrm{t}, J=7.3 \mathrm{~Hz}$, $3 \mathrm{H}) ;{ }^{13} \mathrm{C} \mathrm{NMR}\left(75 \mathrm{MHz}, \mathrm{CDCl}_{3}\right): \delta 193.4,169.0,154.0,136.1,128.9,128.6,127.9,108.1,106.5$, 51.0, 42.4, 32.4, 21.8, 20.5, 13.6; IR (FTIR): $v_{\max } 2963,2933,2873,1962,1770,1711,1441,1416$, 1355, 1146, 732, 699, $628 \mathrm{~cm}^{-1}$; ESIHRMS $\mathrm{m} / \mathrm{z}$ calcd for $\mathrm{C}_{18} \mathrm{H}_{23} \mathrm{~N}_{2} \mathrm{O}_{2}[\mathrm{M}+\mathrm{H}]^{+} 299.1754$, found 299.1761. 


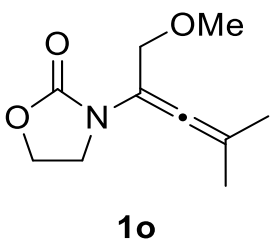

3-(1-Methoxy-4-methylpenta-2,3-dien-2-yl)oxazolidin-2-one 10. Reaction run at $70^{\circ} \mathrm{C}$. Yield: $51 \%$ ( $60.0 \mathrm{mg}, 0.31 \mathrm{mmol}$ ). Solvent system for flash column chromatography: EtOAc/petroleum ether/ $\mathrm{Et}_{3} \mathrm{~N}$ : 25/70/5; Yellow oil; ${ }^{1} \mathrm{H}$ NMR $\left(300 \mathrm{MHz}, \mathrm{CDCl}_{3}\right.$ ): $\delta 4.36-4.21$ (obs. $\mathrm{m}, 2 \mathrm{H}$ ), 4.28 (s, $2 \mathrm{H}), 3.63(\mathrm{t}, J=8.0 \mathrm{~Hz}, 2 \mathrm{H}), 3.31(\mathrm{~s}, 3 \mathrm{H}), 1.77(\mathrm{~s}, 6 \mathrm{H}) ;{ }^{13} \mathrm{C} \mathrm{NMR}\left(75 \mathrm{MHz}, \mathrm{CDCl}_{3}\right): \delta$ 194.8, 155.6, 105.7, 103.8, 70.3, 61.8, 57.6, 46.0, 21.5; IR (FTIR): $v_{\max } 2984,2913,2819,1968,1749,1482,1418$, 1392, 1277, 1243, 1225, 1189, 1120, 1088, 1041, 1012, 947, 915, 981, 757, $730 \mathrm{~cm}^{-1}$; ESIHRMS $m / z$ calcd for $\mathrm{C}_{10} \mathrm{H}_{16} \mathrm{NO}_{3}[\mathrm{M}+\mathrm{H}]^{+} 198.1125$, found 198.1100 .

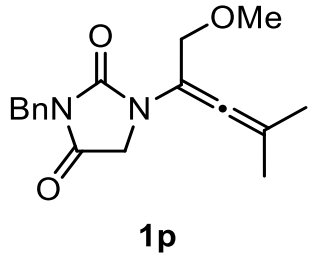

3-Benzyl-1-(1-methoxy-4-methylpenta-2,3-dien-2-yl)imidazolidine-2,4-dione 1p. Yield: 90\% (163.0 mg, $0.54 \mathrm{mmol}$ ). Solvent system for flash column chromatography: EtOAc/petroleum ether/Et $3 \mathrm{~N}$ : 15/80/5; Colorless oil; ${ }^{1} \mathrm{H}$ NMR (300 MHz, $\left.\mathrm{CDCl}_{3}\right): \delta 7.44-7.34(\mathrm{~m}, 2 \mathrm{H}), 7.33-7.22$ (m, 3H), $4.62(\mathrm{~s}, 2 \mathrm{H}), 4.28(\mathrm{~s}, 2 \mathrm{H}), 3.92(\mathrm{~s}, 2 \mathrm{H}), 3.31(\mathrm{~s}, 3 \mathrm{H}), 1.78(\mathrm{~s}, 6 \mathrm{H}) ;{ }^{13} \mathrm{C} \mathrm{NMR}\left(75 \mathrm{MHz}, \mathrm{CDCl}_{3}\right)$ : $\delta$ 194.9, 169.0, 154.0, 136.0, 128.9, 128.6, 127.9, 106.2, 102.9, 70.9, 57.6, 50.7, 42.5, 21.5; IR (FTIR): $v_{\max } 2985,2911,2819,1967,1771,1712,1442,1416,1353,1147,1081,914,730,700$ $\mathrm{cm}^{-1}$; ESIHRMS $\mathrm{m} / \mathrm{z}$ calcd for $\mathrm{C}_{17} \mathrm{H}_{21} \mathrm{~N}_{2} \mathrm{O}_{3}[\mathrm{M}+\mathrm{H}]^{+}$301.1547, found 301.1553. 


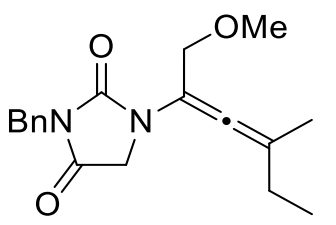

$1 \mathbf{q}$

3-Benzyl-1-(1-methoxy-4-methylhexa-2,3-dien-2-yl)imidazolidine-2,4-dione 1q. Yield: 71\% (134.0 $\mathrm{mg}, 0.43 \mathrm{mmol})$. Solvent system for flash column chromatography: EtOAc/petroleum ether/Et ${ }_{3} \mathrm{~N}$ : 10/85/5; Yellow oil; ${ }^{1} \mathrm{H}$ NMR $\left(300 \mathrm{MHz}, \mathrm{CDCl}_{3}\right): \delta 7.46-7.39(\mathrm{~m}, 2 \mathrm{H}), 7.37-7.27(\mathrm{~m}$, 3H), 4.66 (s, 2H), 4.34 (s, 2H), 3.95 (s, 2H), 3.35 (s, 3H), 2.08 (q, J = 7.4 Hz, 2H), 1.81 (s, 3H), 1.03 $(\mathrm{t}, J=7.4 \mathrm{~Hz}, 3 \mathrm{H}) ;{ }^{13} \mathrm{C} \mathrm{NMR}\left(75 \mathrm{MHz} \mathrm{CDCl}_{3}\right):$ 194.0, 169.1, 153.9, 136.0, 128.9, 128.7, 127.9, 112.5, 105.2, 71.1, 57.7, 50.9, 42.5, 28.0, 20.1, 12.2; IR (FTIR): $v_{\max } 2967,2933,2822,1963,1771,1712$, $1442,1415,1354,1284,1147,1108,1083,911,731,700 \mathrm{~cm}^{-1}$; ESIHRMS m/z calcd for $\mathrm{C}_{18} \mathrm{H}_{23} \mathrm{~N}_{2} \mathrm{O}_{3}$ $[\mathrm{M}+\mathrm{H}]^{+}$315.1703, found 315.1711.

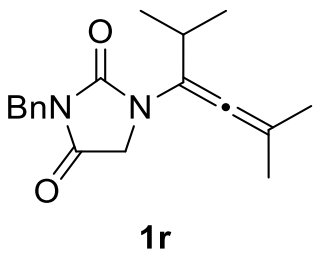

3-Benzyl-1-(2,5-dimethylhexa-3,4-dien-3-yl)imidazolidine-2,4-dione 1r. Yield: 55\% (98.0 mg, $0.33 \mathrm{mmol}$ ). Solvent system for flash column chromatography: EtOAc/petroleum ether/Et $\mathrm{N}_{3}$ : 8/87/5; Yellow oil; ${ }^{1} \mathrm{H}$ NMR (300 MHz, $\left.\mathrm{CDCl}_{3}\right): \delta 7.48-7.40(\mathrm{~m}, 2 \mathrm{H}), 7.38-7.28(\mathrm{~m}, 3 \mathrm{H}), 4.67(\mathrm{~s}$, 2H), $3.90(\mathrm{~s}, 2 \mathrm{H}), 3.20$ (sept., $J=6.7 \mathrm{~Hz}, 1 \mathrm{H}), 1.81(\mathrm{~s}, 6 \mathrm{H}), 1.06(\mathrm{~d}, J=6.8 \mathrm{~Hz}, 6 \mathrm{H}) ;{ }^{13} \mathrm{C} \mathrm{NMR}(75$ $\mathrm{MHz}_{\mathrm{CDCl}}$ ): $\delta 192.9,169.2,154.1,136.2,128.9,128.7,127.9,114.3,107.5,51.6,42.5,28.4,21.7$, 21.4; IR (FTIR): $v_{\max } 2965,2932,2868,1959,1770,1710,1441,1416,1351,1255,1143,727,699$, $623 \mathrm{~cm}^{-1}$; ESIHRMS $\mathrm{m} / z$ calcd for $\mathrm{C}_{18} \mathrm{H}_{23} \mathrm{~N}_{2} \mathrm{O}_{2}[\mathrm{M}+\mathrm{H}]^{+} 299.1754$, found 299.1764 . 


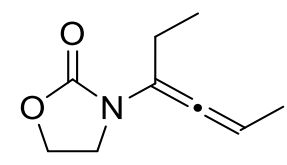

$1 \mathrm{~s}$

3-(Hexa-3,4-dien-3-yl)oxazolidin-2-one 1s. Reaction run at $70^{\circ} \mathrm{C}$. Yield: $45 \%(45.0 \mathrm{mg}, 0.27$ $\mathrm{mmol})$. Solvent system for flash column chromatography: EtOAc/petroleum ether/Et $3 \mathrm{~N}: 20 / 75 / 5$; Yellow oil; ${ }^{1} \mathrm{H}$ NMR (300 MHz, $\mathrm{CDCl}_{3}$ ): $\delta 5.68-5.52$ (br. m, 1H), 4.30 (t, J = 8.0 Hz, 2H), 3.60 (td, J $=7.9$ and $3.3 \mathrm{~Hz}, 2 \mathrm{H}), 2.61-2.46(\mathrm{~m}, 2 \mathrm{H}), 1.71(\mathrm{~d}, J=6.9 \mathrm{~Hz}, 3 \mathrm{H}), 0.98(\mathrm{t}, J=7.3 \mathrm{~Hz}, 3 \mathrm{H}) ;{ }^{13} \mathrm{C} \mathrm{NMR}$ (75 $\mathrm{MHz}_{\mathrm{CDCl}}$ ): $\delta$ 196.9, 155.6, 112.4, 96.5, 61.7, 46.5, 22.5, 16.1, 11.9; IR (FTIR): $v_{\max } 2980$, 2939, 2884, 1963, 1750, 1707, 1391, 1259, 1226, 1121, 1107, 1073, 1039, 982, 938, 916, 760, 731 $\mathrm{cm}^{-1}$; ESIHRMS $\mathrm{m} / \mathrm{z}$ calcd for $\mathrm{C}_{9} \mathrm{H}_{14} \mathrm{NO}_{2}[\mathrm{M}+\mathrm{H}]^{+}$168.1019, found 168.1012.

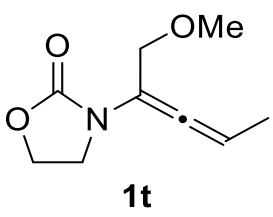

3-(1-Methoxypenta-2,3-dien-2-yl)oxazolidin-2-one 1t. Reaction run at 70 C. Yield: $73 \%(80.0 \mathrm{mg}$, $0.44 \mathrm{mmol}$ ). Solvent system for flash column chromatography: EtOAc/petroleum ether/Et ${ }_{3} \mathrm{~N}$ : 30/65/5; Yellow oil; ${ }^{1} \mathrm{H}$ NMR (300 MHz, $\mathrm{CDCl}_{3}$ ): $\delta 5.72-5.59$ (br. m, $1 \mathrm{H}$ ), $4.39-4.22$ (obs. m, 2H), $4.31(\mathrm{~s}, 2 \mathrm{H}), 3.64(\mathrm{td}, J=7.8$ and $2.2 \mathrm{~Hz}, 2 \mathrm{H}), 3.31(\mathrm{~s}, 3 \mathrm{H}), 1.73(\mathrm{~d}, J=7.0 \mathrm{~Hz}, 3 \mathrm{H}) ;{ }^{13} \mathrm{C}$ NMR $(75$ $\mathrm{MHz}_{\mathrm{CDCl}}$ ): $\delta$ 198.3, 155.4, 106.0, 95.9, 70.0, 61.8, 57.8, 45.9, 15.5; IR (FTIR): $v_{\max } 2983,2921$, 2821, 1967, 1749, 1482, 1425, 1394, 1288, 1255, 1226, 1195, 1117, 1090, 1041, 1015, 908, 756, $732,696 \mathrm{~cm}^{-1}$; ESIHRMS m/z calcd for $\mathrm{C}_{9} \mathrm{H}_{14} \mathrm{NO}_{3}[\mathrm{M}+\mathrm{H}]^{+}$184.0968, found 184.0952 .

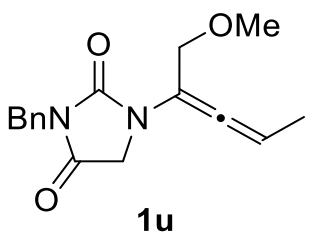

3-Benzyl-1-(1-methoxypenta-2,3-dien-2-yl)imidazolidine-2,4-dione 1u. Yield: 82\% (140.0 mg, $0.49 \mathrm{mmol})$. Solvent system for flash column chromatography: EtOAc/petroleum ether/Et ${ }_{3} \mathrm{~N}$ : 
15/80/5; Yellow oil; ${ }^{1} \mathrm{H}$ NMR (300 MHz, $\left.\mathrm{CDCl}_{3}\right): \delta 7.41-7.41(\mathrm{~m}, 2 \mathrm{H}), 7.39-7.29(\mathrm{~m}, 3 \mathrm{H}), 5.76$ (q, J = $7.0 \mathrm{~Hz}, 1 \mathrm{H}), 4.68(\mathrm{~s}, 2 \mathrm{H}), 4.36(\mathrm{~s}, 2 \mathrm{H}), 4.01(\mathrm{~s}, 2 \mathrm{H}), 3.38(\mathrm{~s}, 3 \mathrm{H}), 1.82(\mathrm{~d}, J=7.1 \mathrm{~Hz}, 3 \mathrm{H}) ;{ }^{13} \mathrm{C}$ $\operatorname{NMR}\left(75 \mathrm{MHz} \mathrm{CDCl}_{3}\right): \delta 198.4,168.9,154.0,135.9,128.9,128.6,127.9,105.0,96.3,70.7,57.8$, 50.6, 42.6, 15.5; IR (FTIR): $v_{\max }$ 2984, 2924, 2822, 1967, 1772, 1712, 1443, 1416, 1355, 1287, 1147, 1106, 1086, 909, 732, $700 \mathrm{~cm}^{-1}$; ESIHRMS $\mathrm{m} / \mathrm{z}$ calcd for $\mathrm{C}_{16} \mathrm{H}_{19} \mathrm{~N}_{2} \mathrm{O}_{3}[\mathrm{M}+\mathrm{H}]^{+} 287.1390$, found 287.1397.

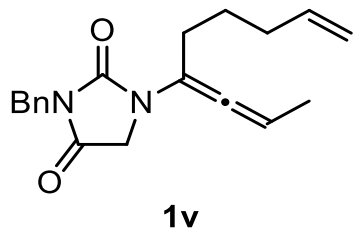

3-Benzyl-1-(nona-2,3,8-trien-4-yl)imidazolidine-2,4-dione 1v. Yield: 73\% (135.0 mg, $0.44 \mathrm{mmol})$. Solvent system for flash column chromatography: EtOAc/petroleum ether/NEt 3 : 5/90/5; Yellow oil; ${ }^{1} \mathrm{H}$ NMR (300 MHz, $\left.\mathrm{CDCl}_{3}\right): \delta 7.56-7.41(\mathrm{~m}, 2 \mathrm{H}), 7.42-7.22(\mathrm{~m}, 3 \mathrm{H}), 5.93-5.76(\mathrm{~m}, 1 \mathrm{H})$, $5.76-5.53(\mathrm{~m}, 1 \mathrm{H}), 5.14-4.93(\mathrm{~m}, 2 \mathrm{H}), 4.69(\mathrm{~s}, 2 \mathrm{H}), 3.93(\mathrm{~d}, J=1.3 \mathrm{~Hz}, 2 \mathrm{H}), 2.69-2.58(\mathrm{~m}, 2 \mathrm{H})$, 2.15 (q, $J=7.3 \mathrm{~Hz}, 2 \mathrm{H}), 1.78$ (d, $J=7.0 \mathrm{~Hz}, 3 \mathrm{H}$ ), 1.58 (app. quint., $J=7.2 \mathrm{~Hz}, 2 \mathrm{H}) ;{ }^{13} \mathrm{C} \mathrm{NMR}(75 \mathrm{MHz}$, $\left.\mathrm{CDCl}_{3}\right): \delta 197.3,168.8,154.0,138.4,136.1,128.9,128.7,128.0,114.9,110.1$, 96.9, 51.0, 42.5, 33.1, 29.5, 26.6, 16.0; IR (FTIR): $v_{\max } 3066,3033,2974,2935,1959,1767,1708,1439,1414,1356$, $1269,1255,1145,899,736,695 \mathrm{~cm}^{-1}$; ESIHRMS $m / z$ calcd for $\mathrm{C}_{19} \mathrm{H}_{23} \mathrm{~N}_{2} \mathrm{O}_{2}[\mathrm{M}+\mathrm{H}]^{+} 311.1754$, found 311.1759 .

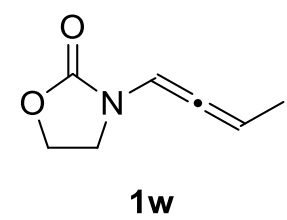

3-(Buta-1,2-dien-1-yl)oxazolidin-2-one 1w. ${ }^{53}$ Yield: $46 \%$ (39.0 mg, $\left.0.25 \mathrm{mmol}\right)$. Solvent system for flash column chromatography: EtOAc/petroleum ether/Et ${ }_{3} \mathrm{~N}$ : 15/80/5; Orange oil; ${ }^{1} \mathrm{H}$ NMR (300 MHz, $\mathrm{CDCl}_{3}$ ): $\delta 6.80-6.70(\mathrm{~m}, 1 \mathrm{H}), 5.79$ (quint., $\left.J=6.6 \mathrm{~Hz}, 1 \mathrm{H}\right), 4.40(\mathrm{t}, J=8.0 \mathrm{~Hz}, 2 \mathrm{H}), 3.67$ - $3.43(\mathrm{~m}, 2 \mathrm{H}), 1.77(\mathrm{dd}, J=6.6$ and $2.1 \mathrm{~Hz}, 3 \mathrm{H}) ;{ }^{13} \mathrm{C} \mathrm{NMR}\left(75 \mathrm{MHz}, \mathrm{CDCl}_{3}\right): \delta 195.6,155.5,99.0$, 
96.3, 62.3, 43.3, 16.5; IR (ATR): $v_{\max } 2983,2918,1972,1748,1484,1467,1438,1394,1280,1234$, $1078,1060,1031,875,753,670 \mathrm{~cm}^{-1}$; ESIHRMS m/z calcd for $\mathrm{C}_{7} \mathrm{H}_{10} \mathrm{NO}_{2}[\mathrm{M}+\mathrm{H}]^{+}$140.0706, found 140.0705 .

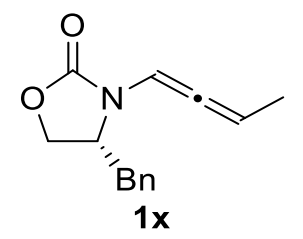

(4R)-4-Benzyl-3-(buta-1,2-dien-1-yl)oxazolidin-2-one $1 x^{\mathrm{S3}}$ Obtained as a ca 1:1 mixture of diastereoisomers. Yield: $43 \%(59.0 \mathrm{mg}, 0.26 \mathrm{mmol})$. Solvent system for flash column chromatography: EtOAc/petroleum ether/Et $3 \mathrm{~N}$ : 15/80/5; Amorphous orange solid; ${ }^{1 \mathrm{H}} \mathrm{NMR}$ (300 $\mathrm{MHz}_{\mathrm{CDCl}}$ ): $\delta 7.40-7.20(\mathrm{~m}, 3 \mathrm{H}), 7.20-7.10(\mathrm{~m}, 2 \mathrm{H}), 6.84-6.73$ (br. $\left.\mathrm{m}, 1 \mathrm{H}\right), 6.01-5.80(\mathrm{~m}$, $1 \mathrm{H}), 4.30-4.18(\mathrm{~m}, 1 \mathrm{H}), 4.18-4.00(\mathrm{~m}, 2 \mathrm{H}), 3.31-3.10(\mathrm{~m}, 1 \mathrm{H}), 2.68(\mathrm{~m}, 1 \mathrm{H}), 1.86$ and 1.85 (app. t, $J=6.9 \mathrm{~Hz}, 1.5 \mathrm{H}$, app. t, $J=6.9 \mathrm{~Hz}, 1.5 \mathrm{H}$, diastereoisomers); ${ }^{13} \mathrm{C} \mathrm{NMR}\left(75 \mathrm{MHz}, \mathrm{CDCl}_{3}\right): \delta$ 196.3 and 195.8 (diastereoisomers), 155.2 and 155.1 (diastereoisomers), 135.7, 129.4 and 129.3 (diastereoisomers), 129.1 and 129.0 (diastereoisomers), 127.4 and 127.3 (diastereoisomers), 99.1 and 98.8 (diastereoisomers), 95.3 and 95.1 (diastereoisomers), 66.7 and 66.6 (diastereoisomers), 55.9 and 55.7 (diastereoisomers), 37.5 and 36.5 (diastereoisomers), 16.4 and 16.2 (diastereoisomers); IR (ATR): $v_{\max }$ 3030, 2916, 1970, 1752, 1468, 1435, 1401, 1260, 1234, 1082, 876, 741, 704, $682 \mathrm{~cm}^{-1}$; ESIHRMS $\mathrm{m} / \mathrm{z}$ calcd for $\mathrm{C}_{14} \mathrm{H}_{16} \mathrm{NO}_{2}[\mathrm{M}+\mathrm{H}]^{+} 230.1176$, found 230.1178 .

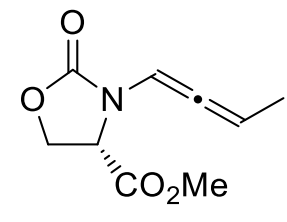

$1 y$

Methyl (4S)-3-(buta-1,2-dien-1-yl)oxazolidin-2-one-4-carboxylate 1y. Obtained as a ca 1:1 mixture of diastereoisomers. Yield: $57 \%(67.0 \mathrm{mg}, 0.34 \mathrm{mmol})$. Solvent system for flash column chromatography: EtOAc/petroleum ether/Et ${ }_{3} \mathrm{~N}$ : 7.5/90/2.5; Yellow oil; ${ }^{1} \mathrm{H} \mathrm{NMR}\left(300 \mathrm{MHz}, \mathrm{CDCl}_{3}\right)$ : 
$\delta 6.75-6.61(\mathrm{~m}, J=2.8 \mathrm{~Hz}, 1 \mathrm{H}$ ), 5.74 (app. quint., $J=6.7 \mathrm{~Hz}, 1 \mathrm{H}$ ), 4.56 (A of ABX syst., $J=9.0 \mathrm{~Hz}$ and $9.0 \mathrm{~Hz}, 1 \mathrm{H}), 4.45-4.24(\mathrm{~m}, 2 \mathrm{H}), 3.76$ and $3.75(\mathrm{~s}, 1.5 \mathrm{H}, \mathrm{s}, 1.5 \mathrm{H}$, diastereoisomers), 1.72 and $1.65\left(\mathrm{dd}, J=6.9\right.$ and $2.6 \mathrm{~Hz}, 1.5 \mathrm{H}$, dd, $J=6.9$ and $2.6 \mathrm{~Hz}, 1.5 \mathrm{H}$, diastereoisomers); ${ }^{13} \mathrm{C}$ NMR $(75$ $\mathrm{MHz}, \mathrm{CDCl}_{3}$ ): $\delta 195.3$ and 195.1 (diastereoisomers), 169.9 and 169.5 (diastereoisomers), 154.6 and 154.6 (diastereoisomers), 100.3 and 100.1 (diastereoisomers), 95.4, 65.0 and 65.0 (diastereoisomers), 56.6 and 56.4 (diastereoisomers), 53.1 and 52.8 (diastereoisomers), 16.3 and 16.1 (diastereoisomers); IR (FTIR): $v_{\max }$ 3043, 2956, 2919, 1974, 1754, 1467, 1435, 1398, 1328, 1210, 1082, 1061, 992, 873, 753, $688 \mathrm{~cm}^{-1}$; ESIHRMS m/z calcd for $\mathrm{C}_{9} \mathrm{H}_{12} \mathrm{NO}_{4}[\mathrm{M}+\mathrm{H}]^{+} 198.0761$, found 198.0760 .

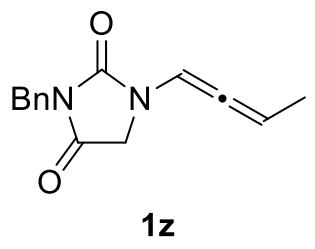

3-Benzyl-1-(buta-1,2-dien-1-yl)imidazolidine-2,4-dione 1z. Yields: 74\% (107.0 mg, 0.44 mmol; reaction performed on a $0.6 \mathrm{mmol}$ scale) and $60 \%(1.15 \mathrm{~g}, 4.8 \mathrm{mmol}$; reaction performed on a $8.0 \mathrm{mmol}$ scale). Solvent system for flash column chromatography: EtOAc/petroleum ether/Et ${ }_{3} \mathrm{~N}$ : 20/77.5/2.5; Yellow solid; Mp: $69^{\circ} \mathrm{C} ;{ }^{1} \mathrm{H}$ NMR (300 MHz, $\left.\mathrm{CDCl}_{3}\right): \delta 7.45-7.37$ (m, 2H), $7.37-7.27$ (m, 3H), 6.93 (sext., $J=2.9 \mathrm{~Hz}, 1 \mathrm{H}$ ), 5.84 (quint., $J=6.8 \mathrm{~Hz}, 1 \mathrm{H}), 4.68(\mathrm{~s}, 2 \mathrm{H}$ ), 3.86 (d app., $J=1.6$ $\mathrm{Hz}, 2 \mathrm{H}) 1.77$ (app. dd, $J=7.0$ and $2.7 \mathrm{~Hz}, 3 \mathrm{H}) ;{ }^{13} \mathrm{C} \mathrm{NMR}\left(75 \mathrm{MHz}, \mathrm{CDCl}_{3}\right.$ ): $\delta$ 195.4, 168.8, 153.9, 135.9, 129.0, 128.8, 128.2, 100.1, 94.6, 48.7, 42.8, 16.4; IR (FTIR): $v_{\max } 2981,2924,1970,1773$, 1711, 1476, 1449, 1434, 1408, 1354, 1272, 1146, 980, 744, 730, $700 \mathrm{~cm}^{-1}$; ESIHRMS m/z calcd for $\mathrm{C}_{14} \mathrm{H}_{15} \mathrm{~N}_{2} \mathrm{O}_{2}[\mathrm{M}+\mathrm{H}]^{+}$243.1128, found 243.1133. 
Supporting Information

\section{${ }^{1} \mathrm{H}$ and ${ }^{13} \mathrm{C}$ NMR spectra}



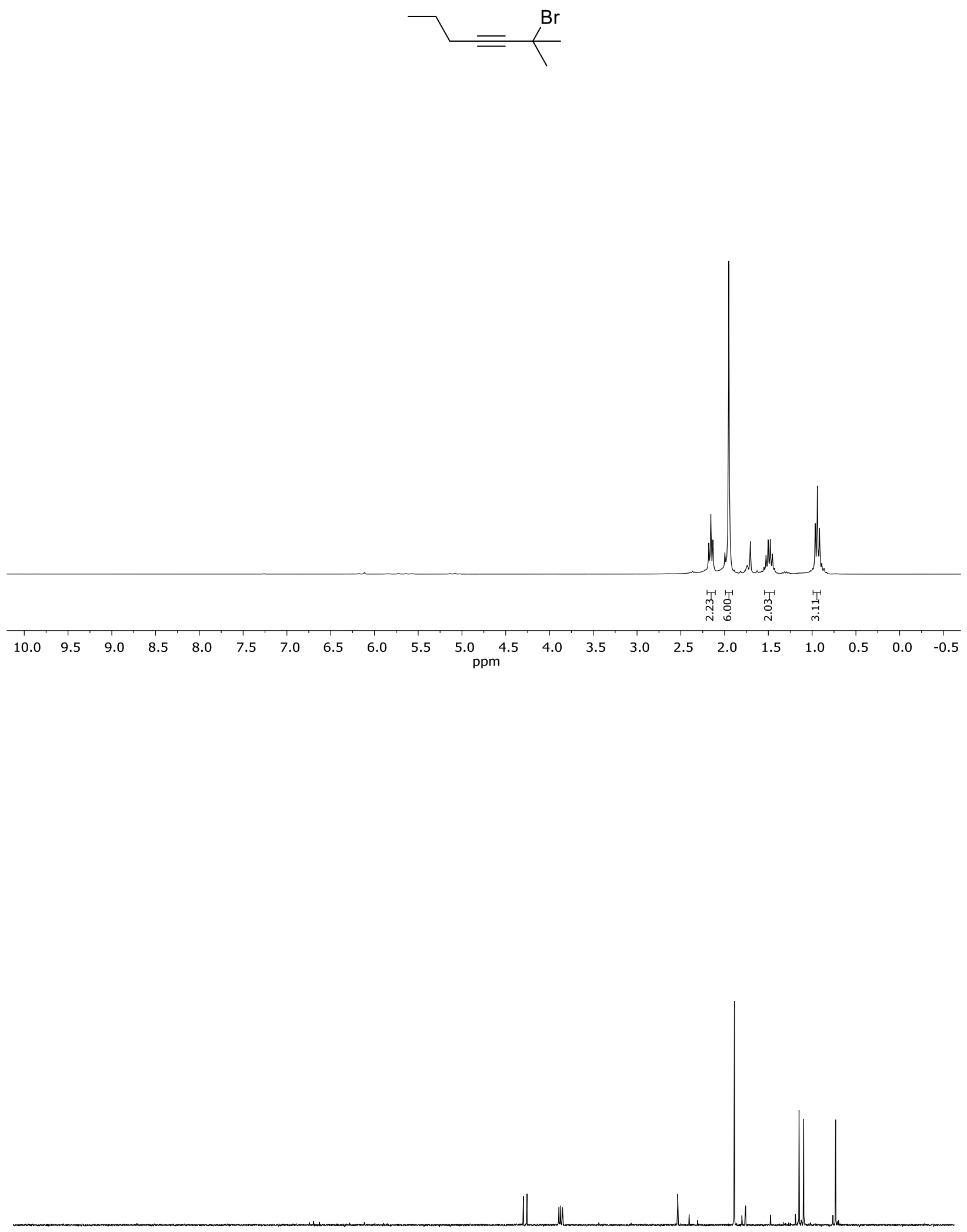

$\begin{array}{lllllllllllllllllllllll}200 & 190 & 180 & 170 & 160 & 150 & 140 & 130 & 120 & 110 & 100 & 90 & 80 & 70 & 60 & 50 & 40 & 30 & 20 & 10 & 0 & -10\end{array}$ 

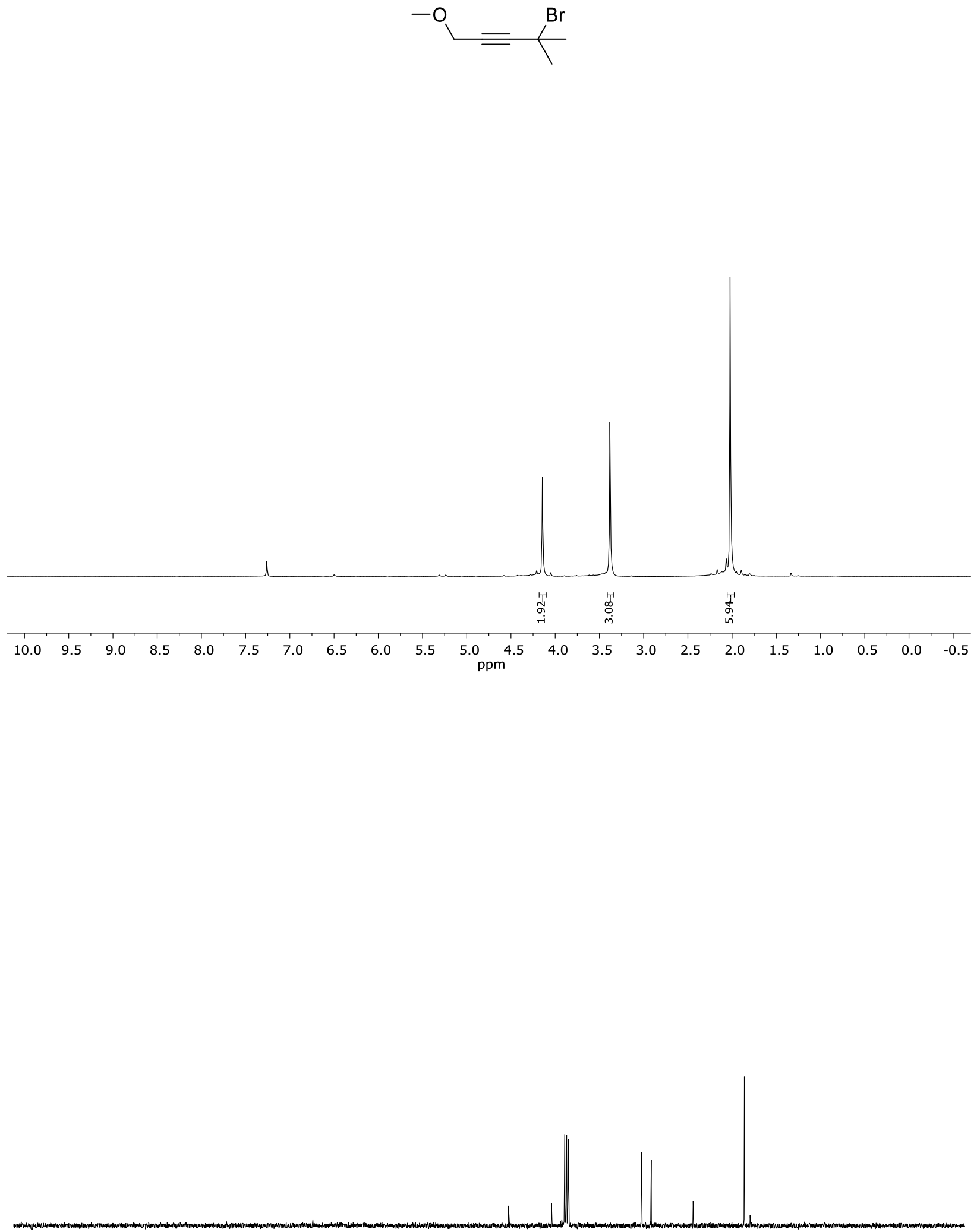

$\begin{array}{llllllllllllllllllllllll}200 & 190 & 180 & 170 & 160 & 150 & 140 & 130 & 120 & 110 & 100 & 90 & 80 & 70 & 60 & 50 & 40 & 30 & 20 & 10 & 0 & -10\end{array}$



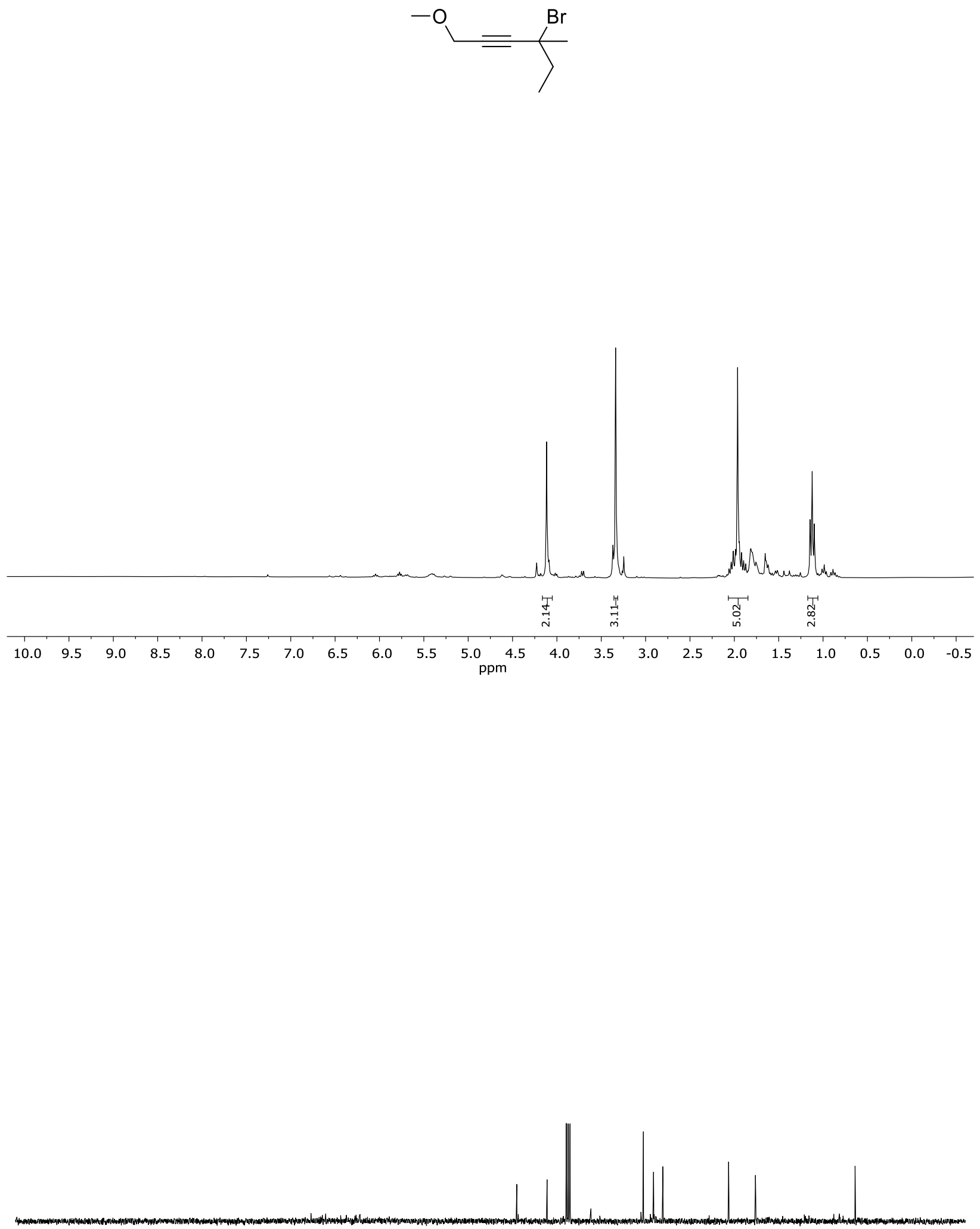

$\begin{array}{lllllllllllllllllllllllll}200 & 190 & 180 & 170 & 160 & 150 & 140 & 130 & 120 & 110 & 100 & 90 & 80 & 70 & 60 & 50 & 40 & 30 & 20 & 10 & 0 & -10\end{array}$



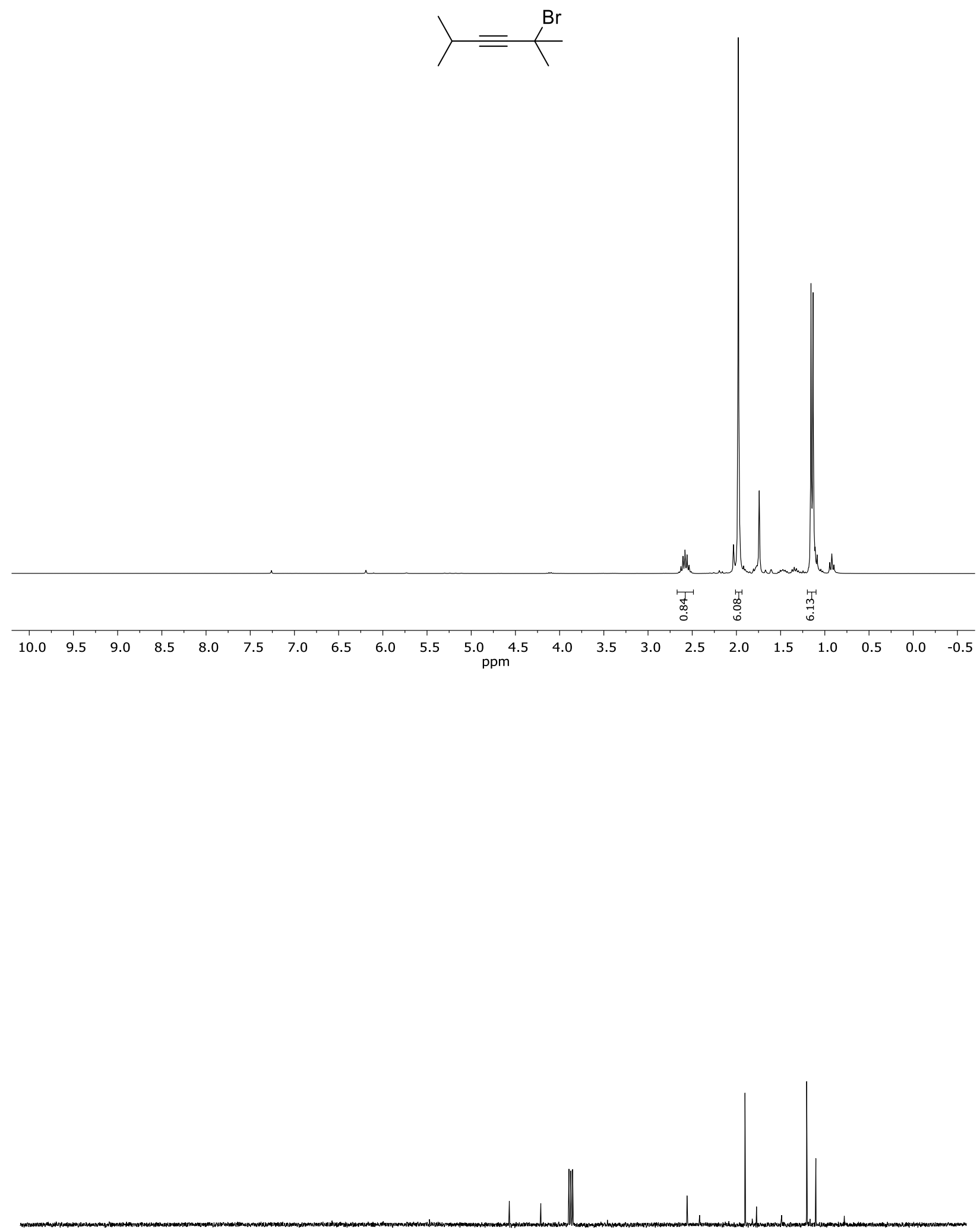

$\begin{array}{lllllllllllllllllllllllll}200 & 190 & 180 & 170 & 160 & 150 & 140 & 130 & 120 & 110 & 100 & 90 & 80 & 70 & 60 & 50 & 40 & 30 & 20 & 10 & 0 & -10\end{array}$



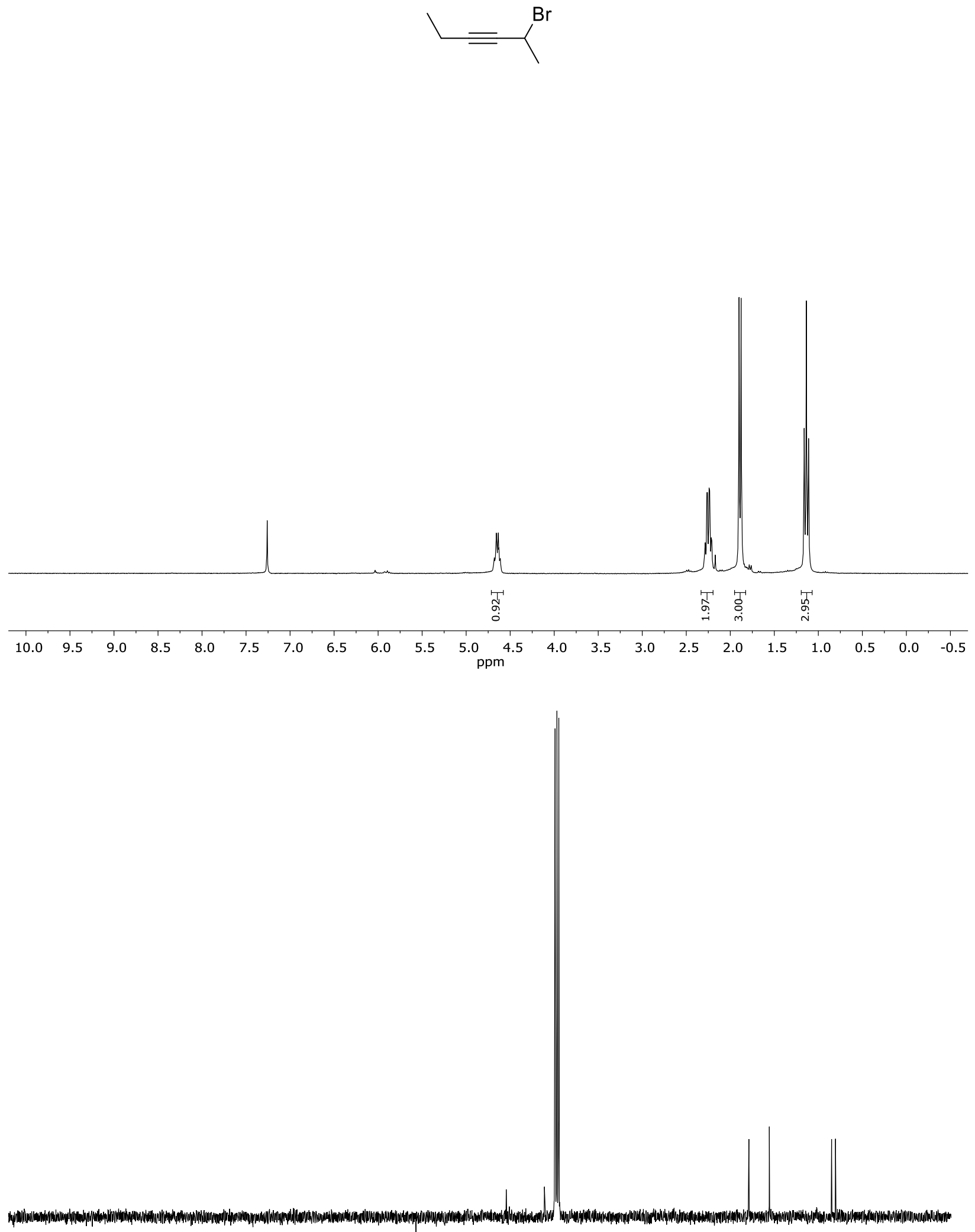

$\begin{array}{lllllllllllllllllllllll}200 & 190 & 180 & 170 & 160 & 150 & 140 & 130 & 120 & 110 & 100 & 90 & 80 & 70 & 60 & 50 & 40 & 30 & 20 & 10 & 0 & -10\end{array}$ 

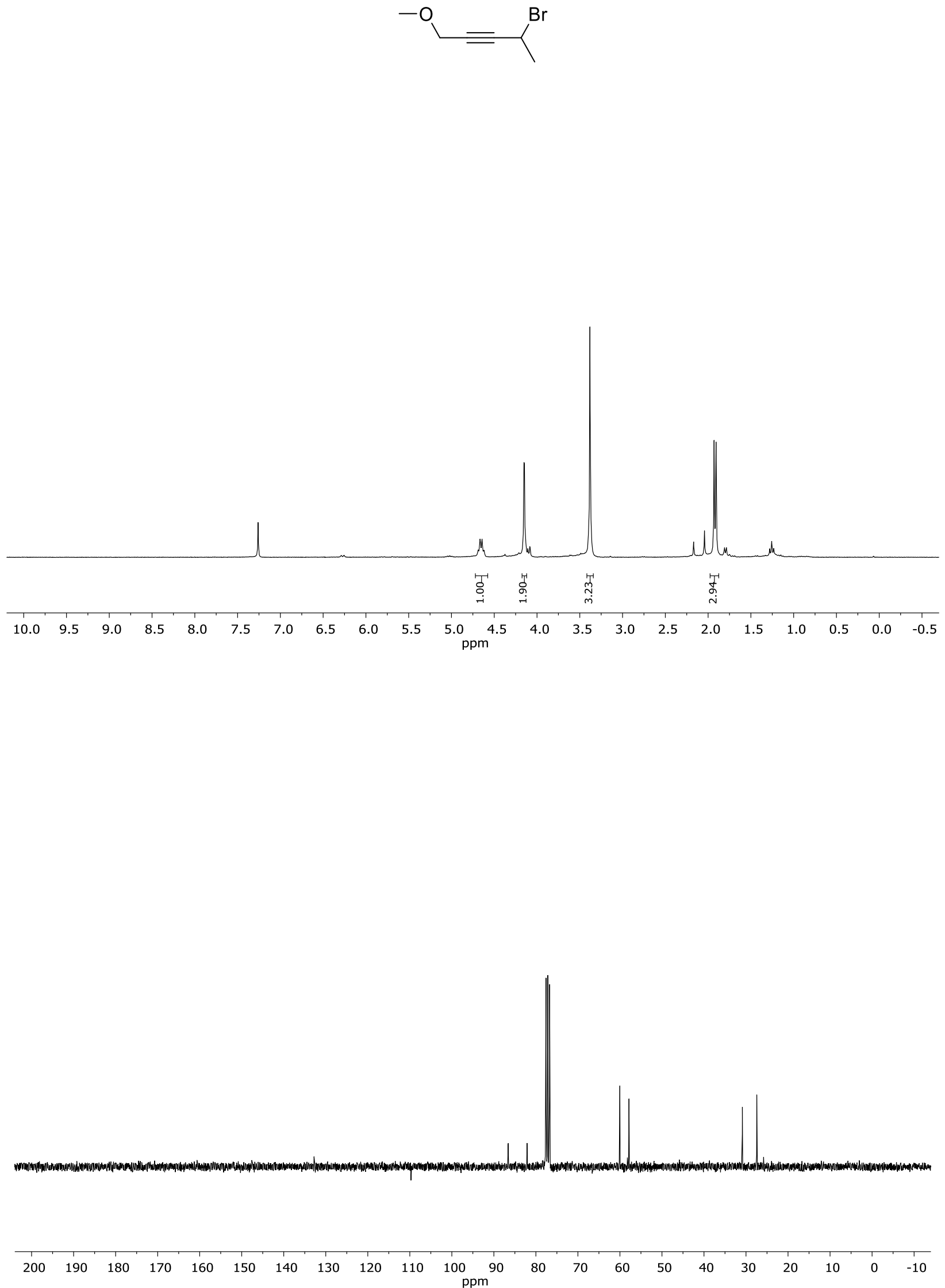

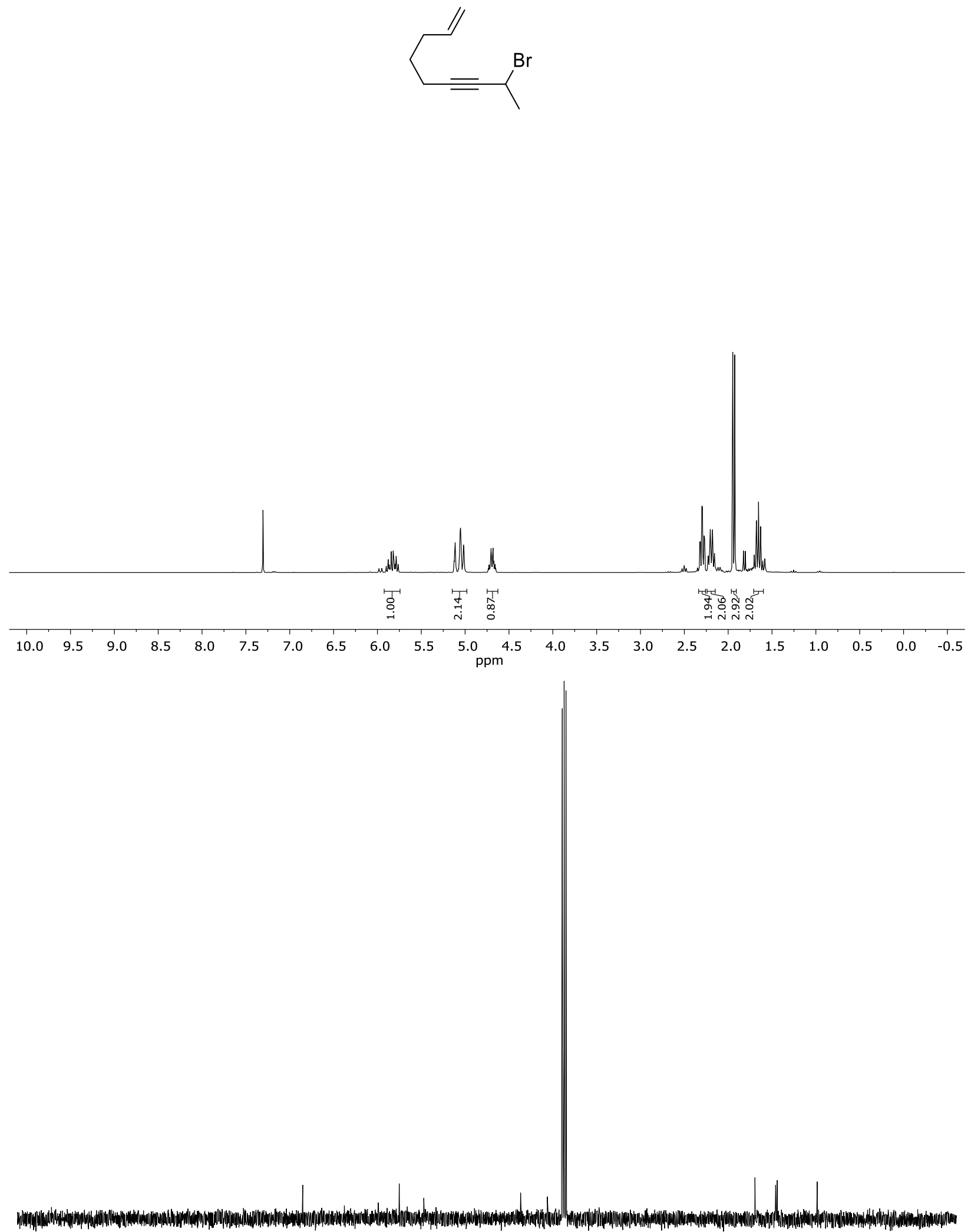

\begin{tabular}{lllllllllllllllllllllll}
\hline & 190 & 180 & 170 & 160 & 150 & 140 & 130 & 120 & 110 & 100 & 90 & 80 & 70 & 60 & 50 & 40 & 30 & 20 & 10 & 0 & -10
\end{tabular}




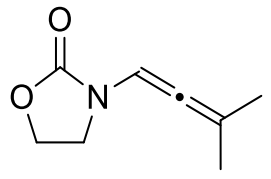

$1 a$
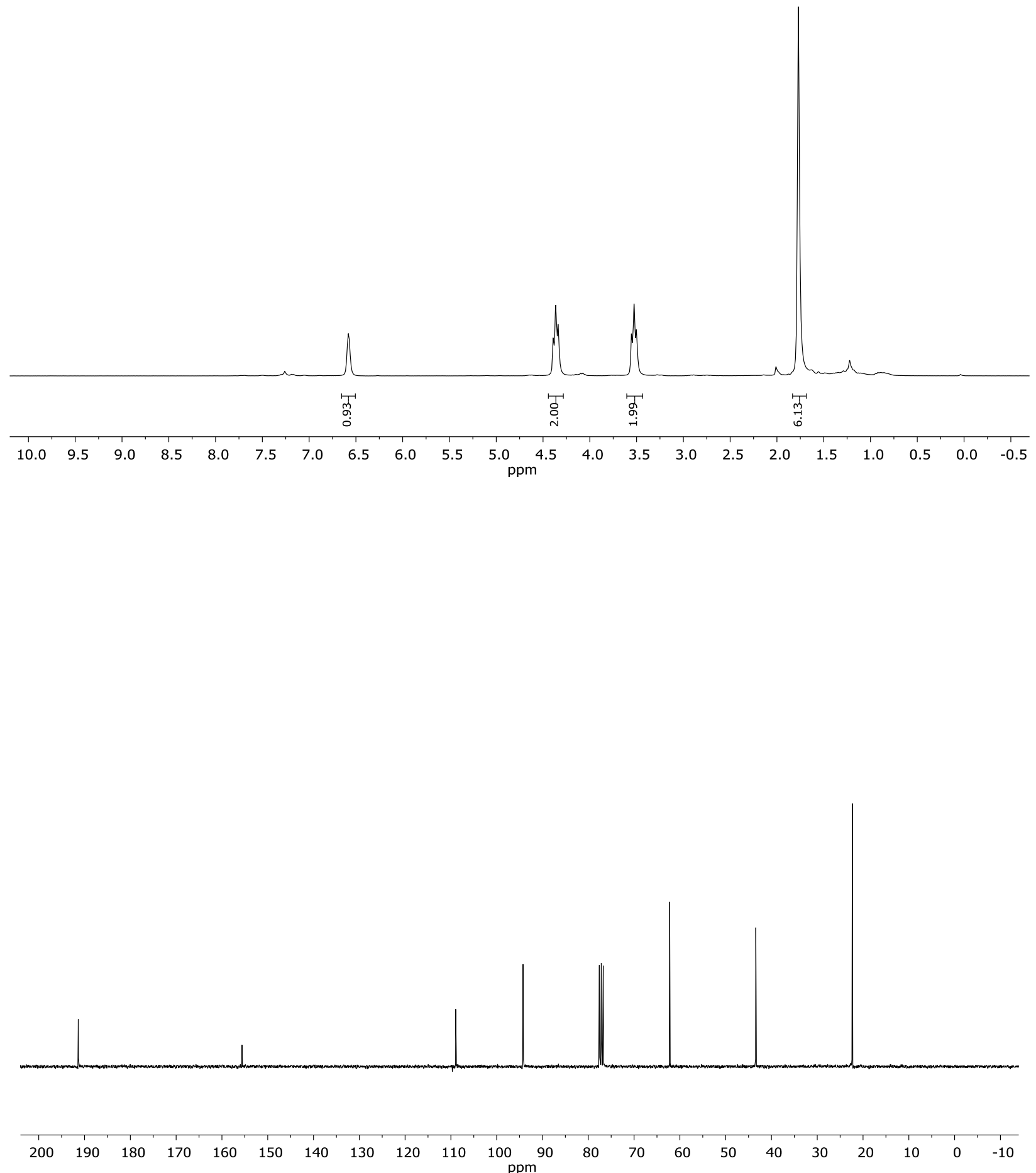


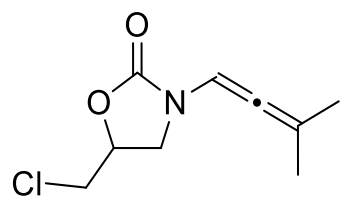

$1 b$
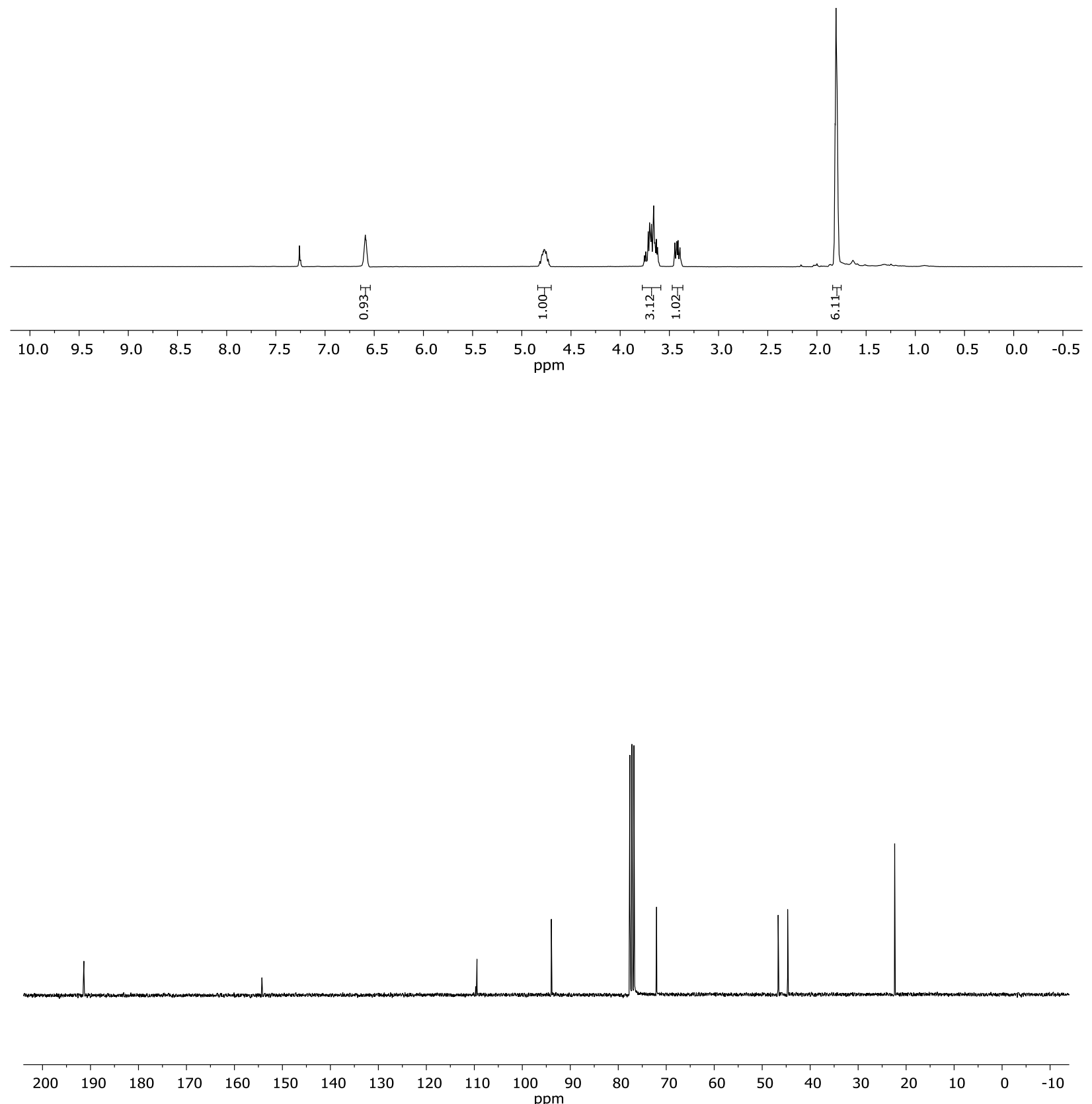


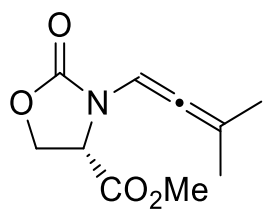

$1 \mathrm{c}$
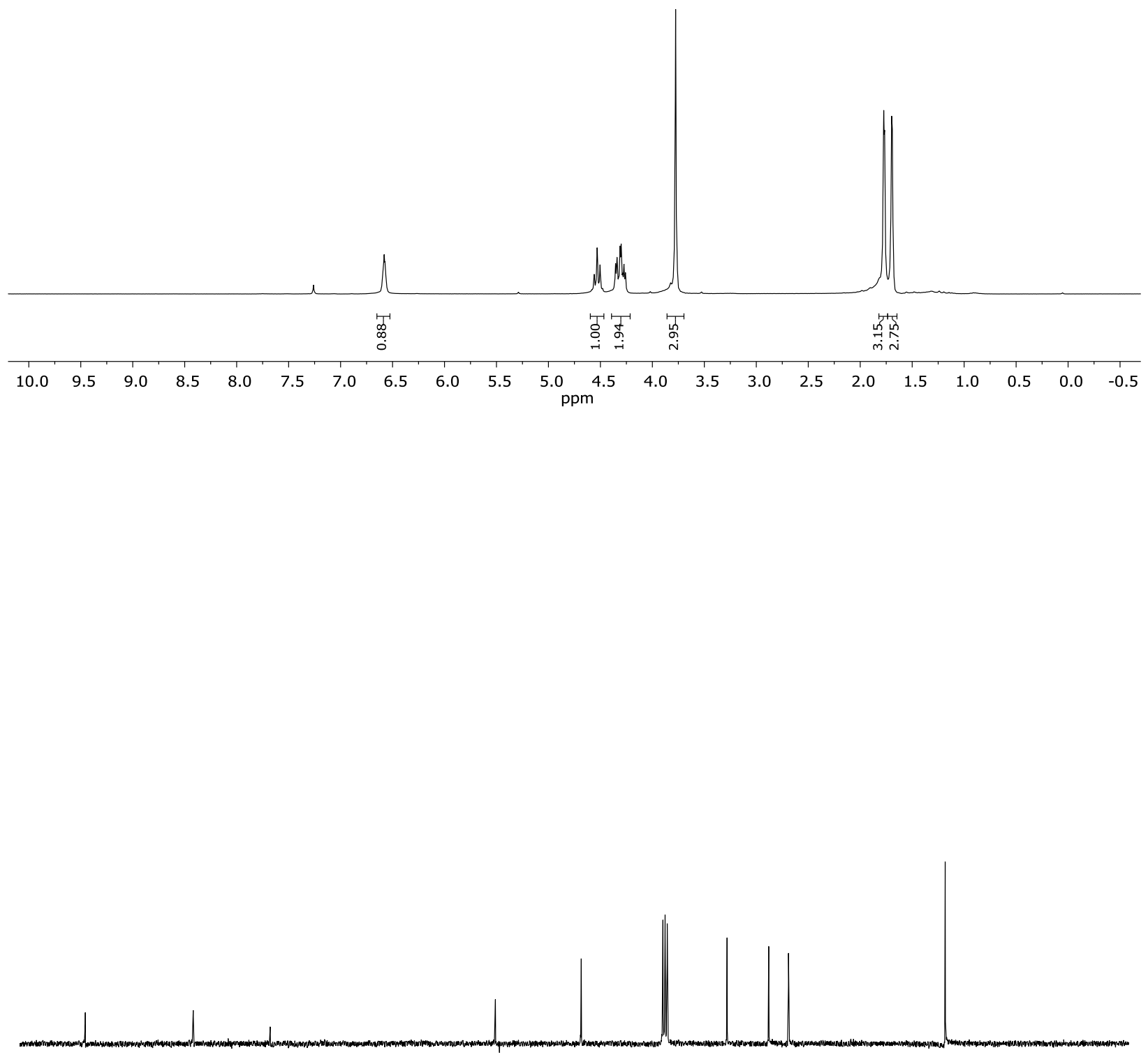

$\begin{array}{lllllllllllllllllllllllllll}200 & 190 & 180 & 170 & 160 & 150 & 140 & 130 & 120 & 110 & 100 & 90 & 80 & 70 & 60 & 50 & 40 & 30 & 20 & 10 & 0 & -10\end{array}$




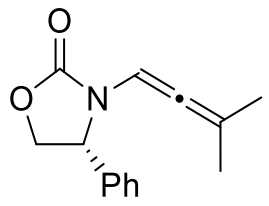

$1 d$
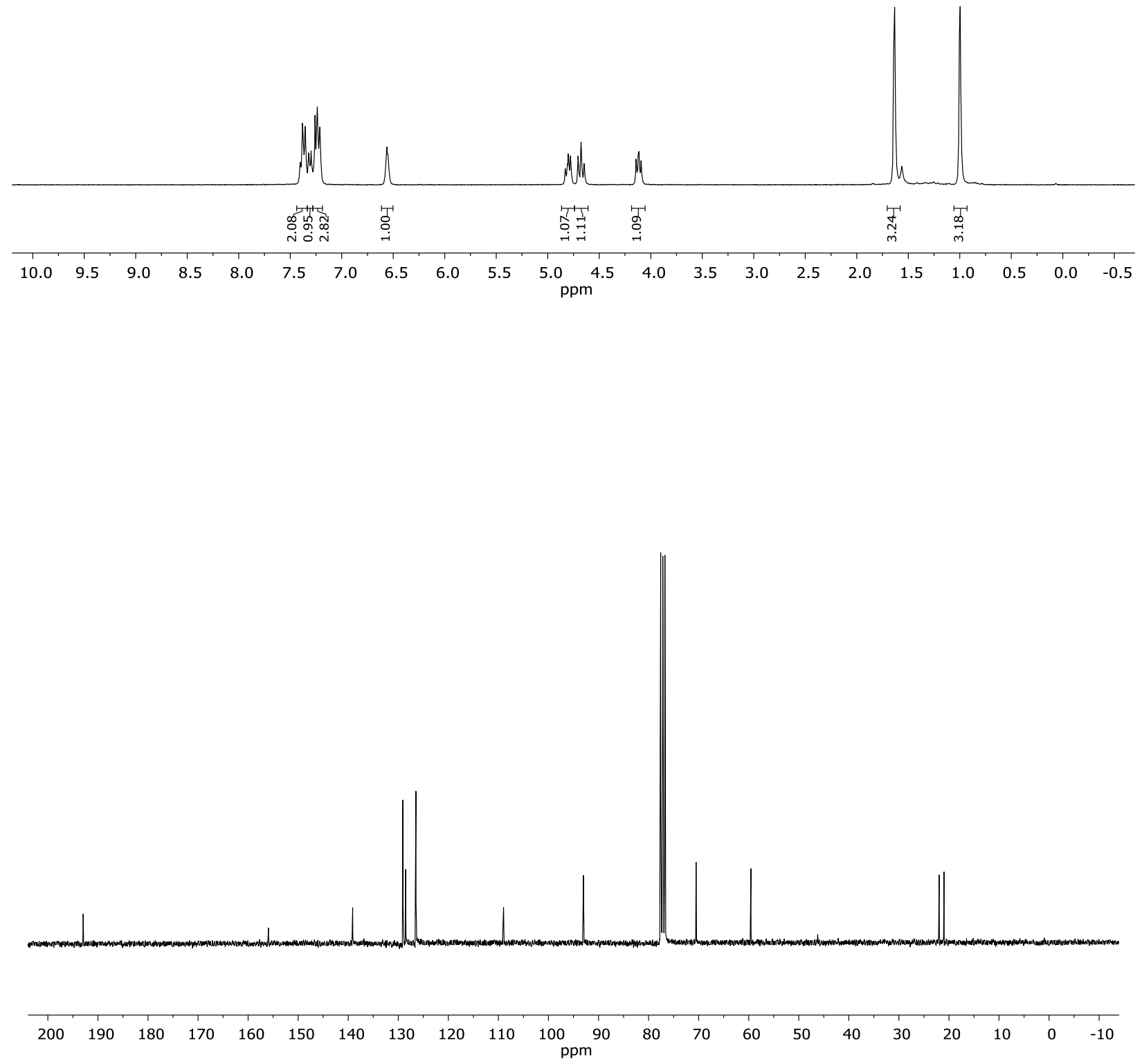


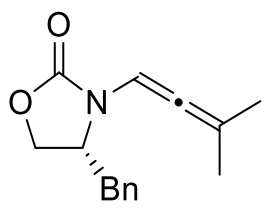

$1 e$
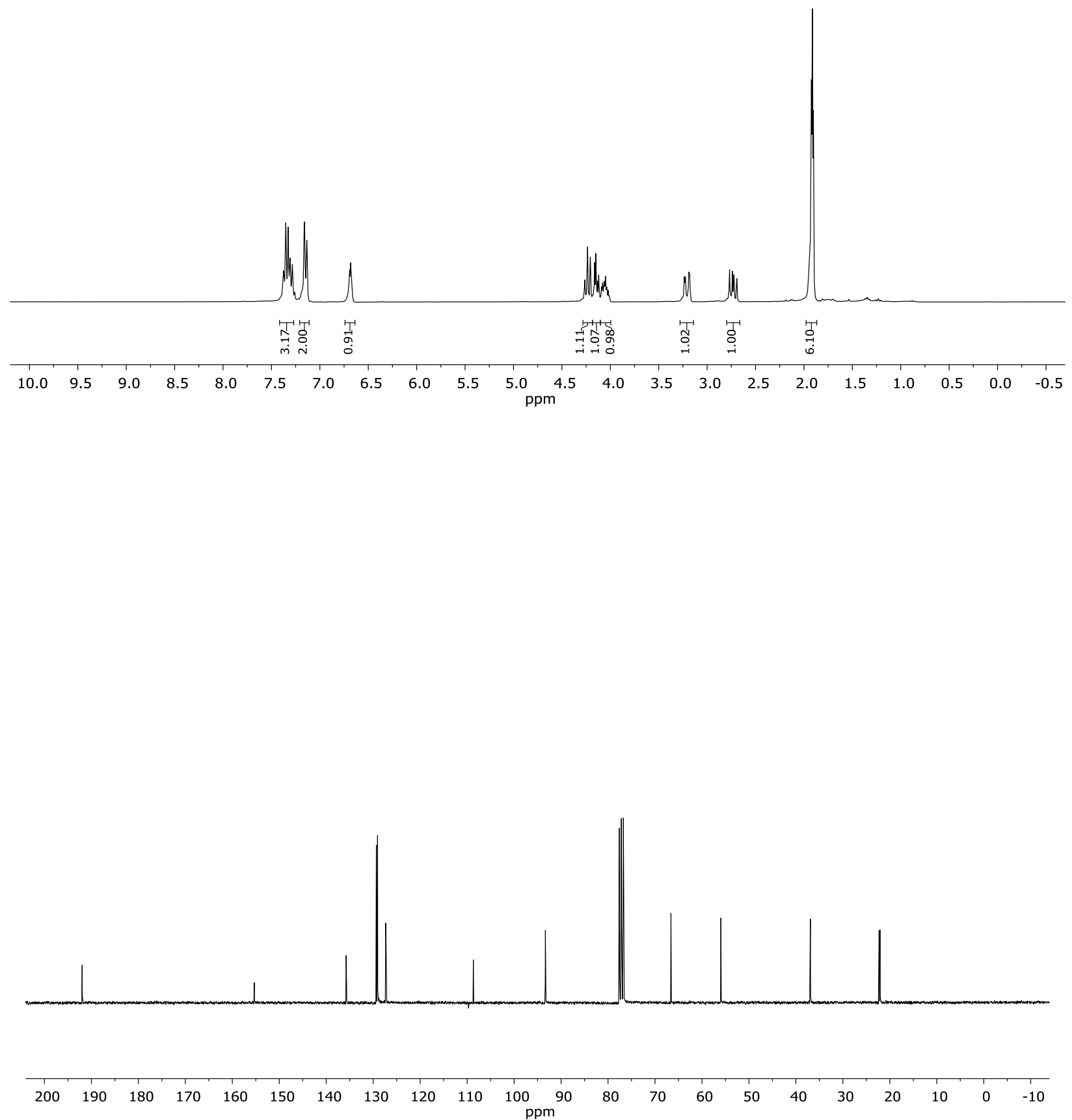


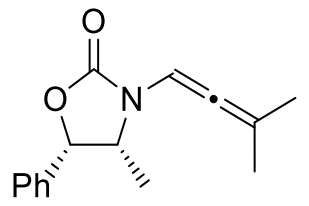

$1 f$
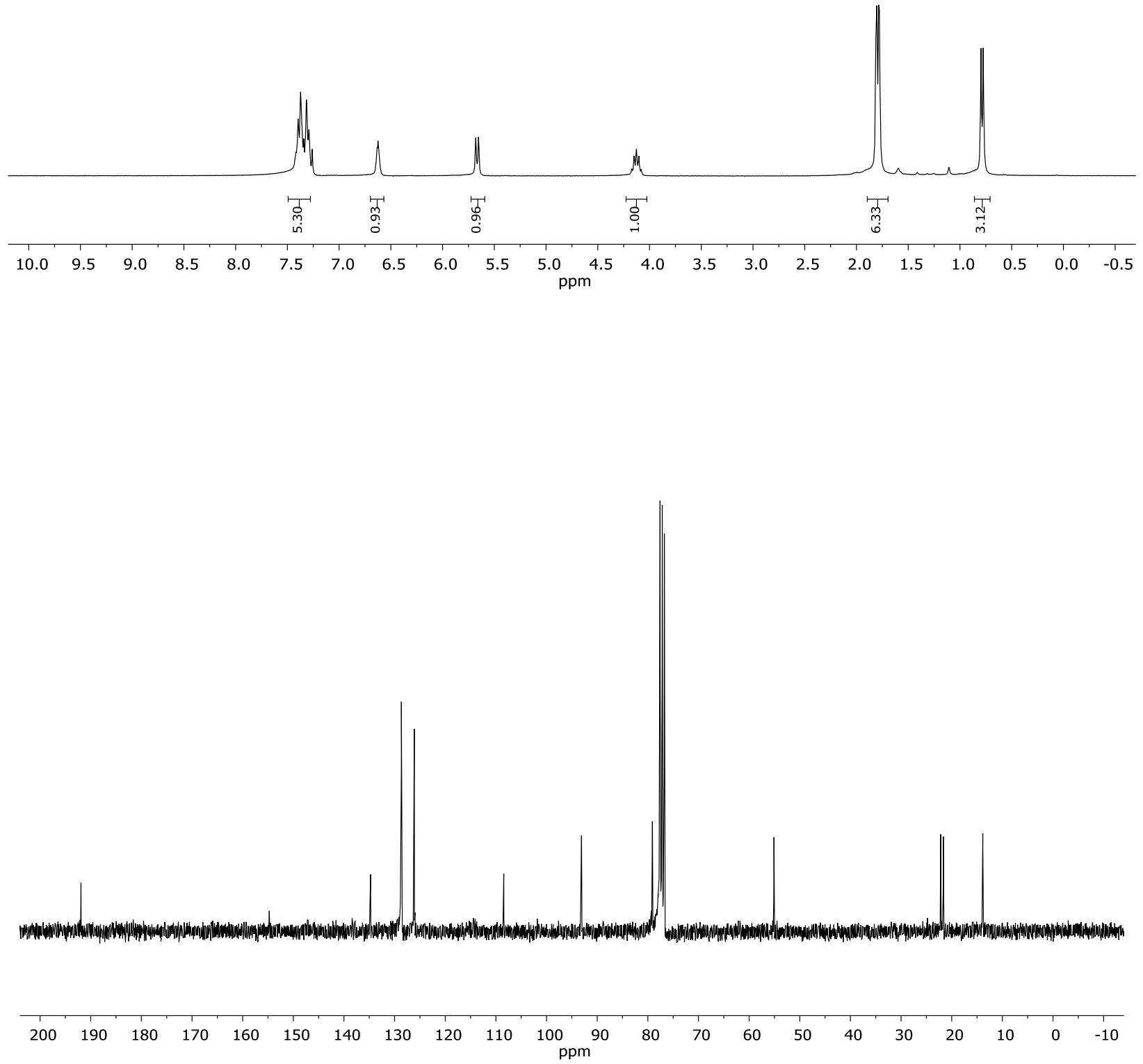


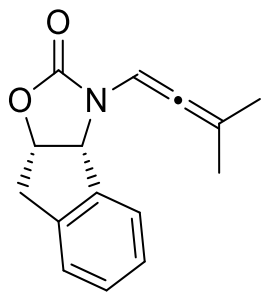

$1 \mathrm{~g}$
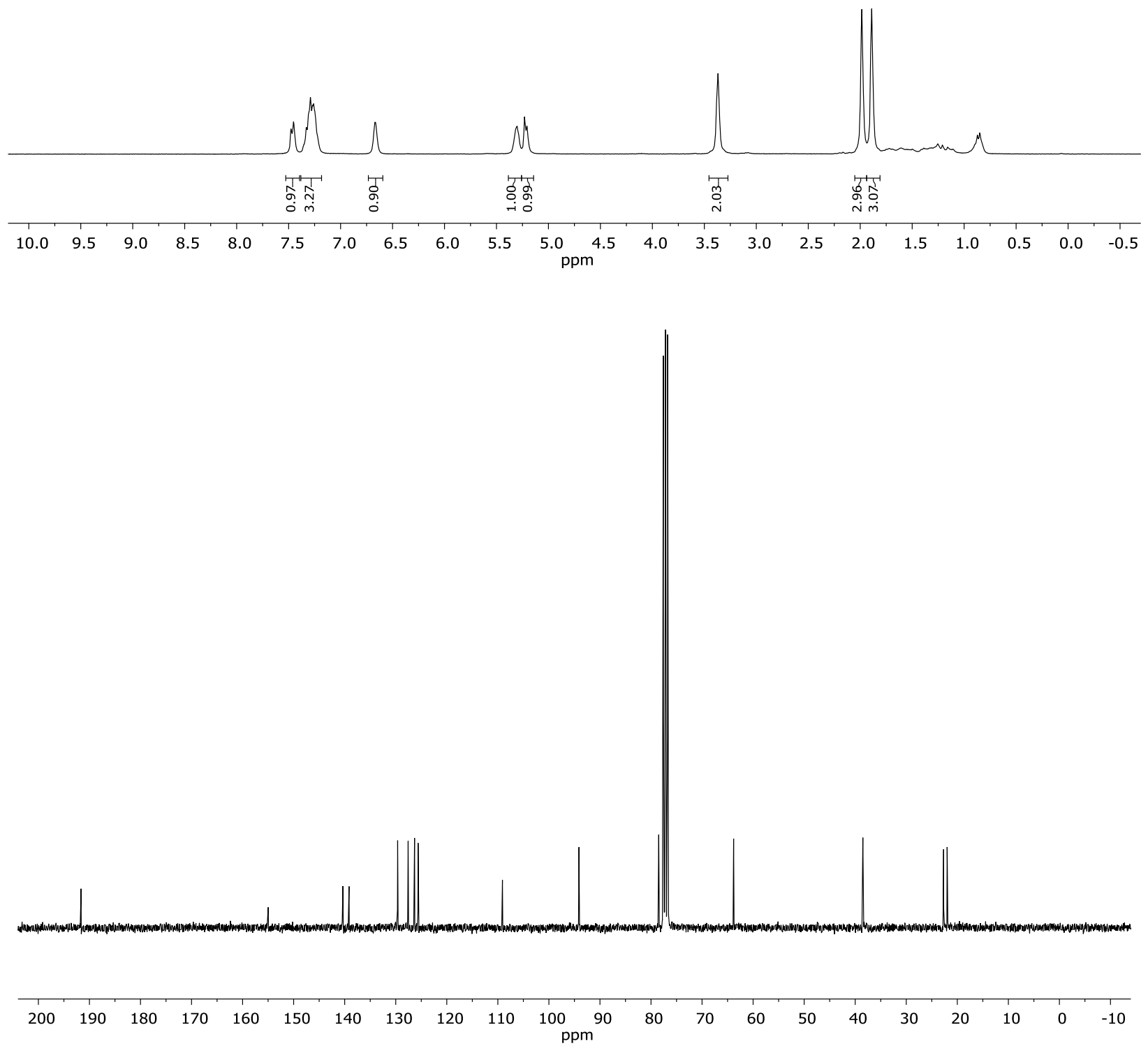


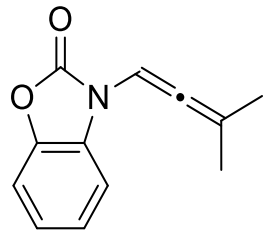

$1 \mathrm{~h}$
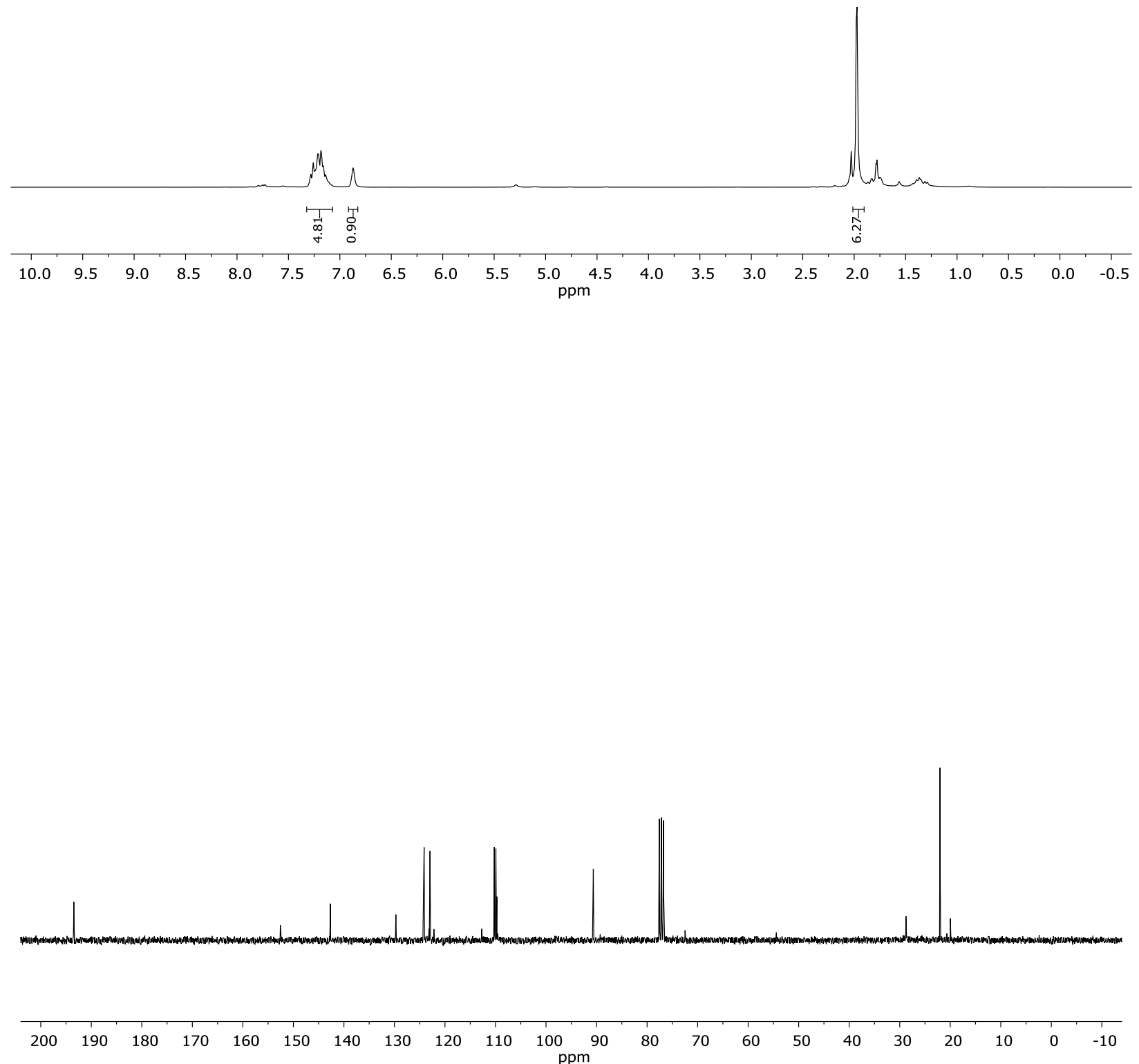


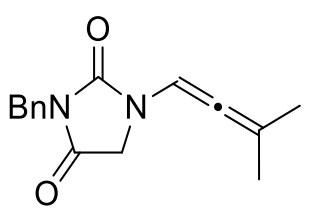

$1 \mathrm{i}$
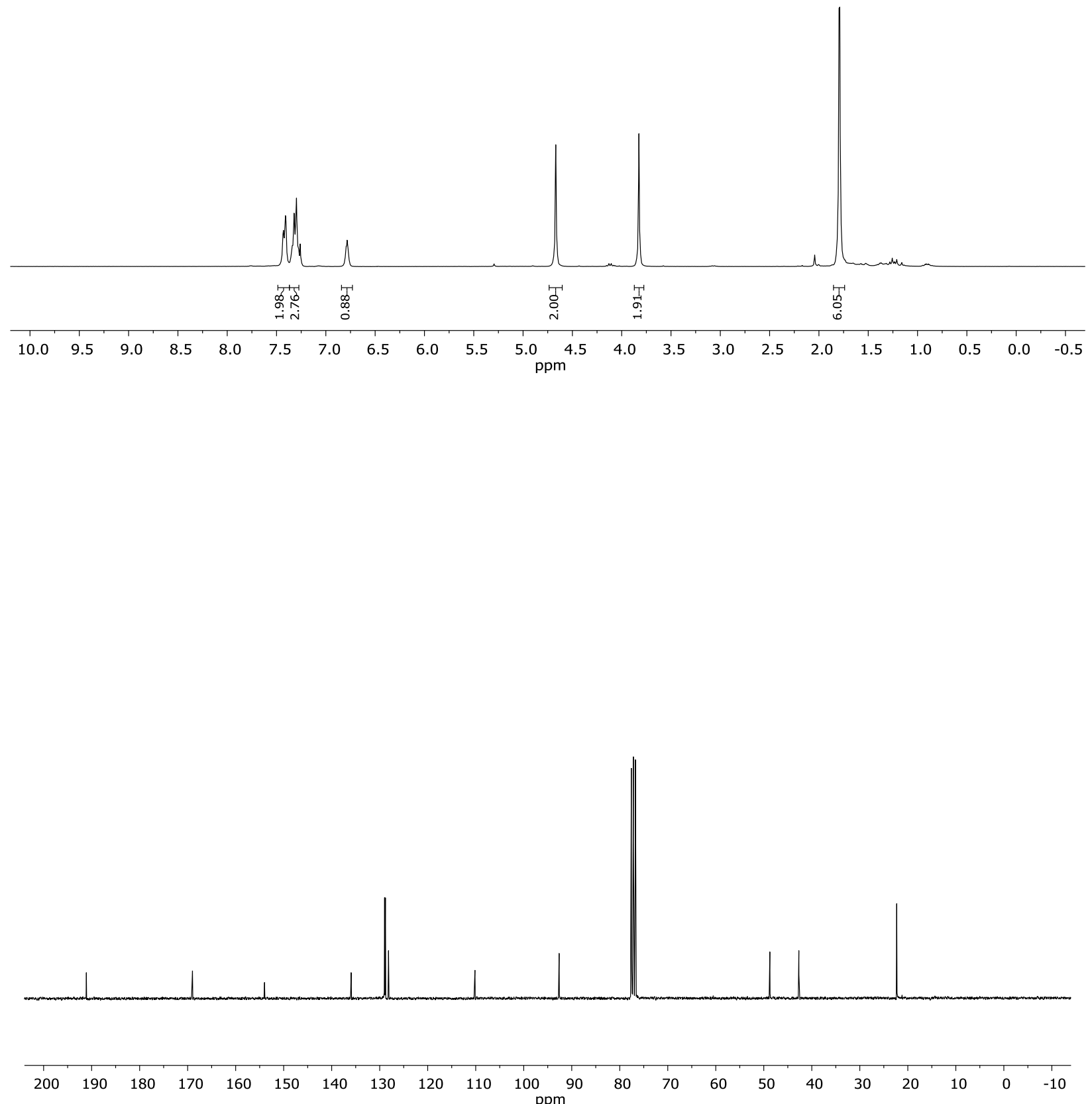


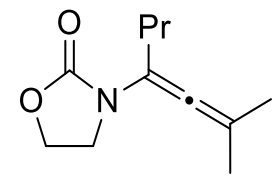

$1 \mathrm{~m}$
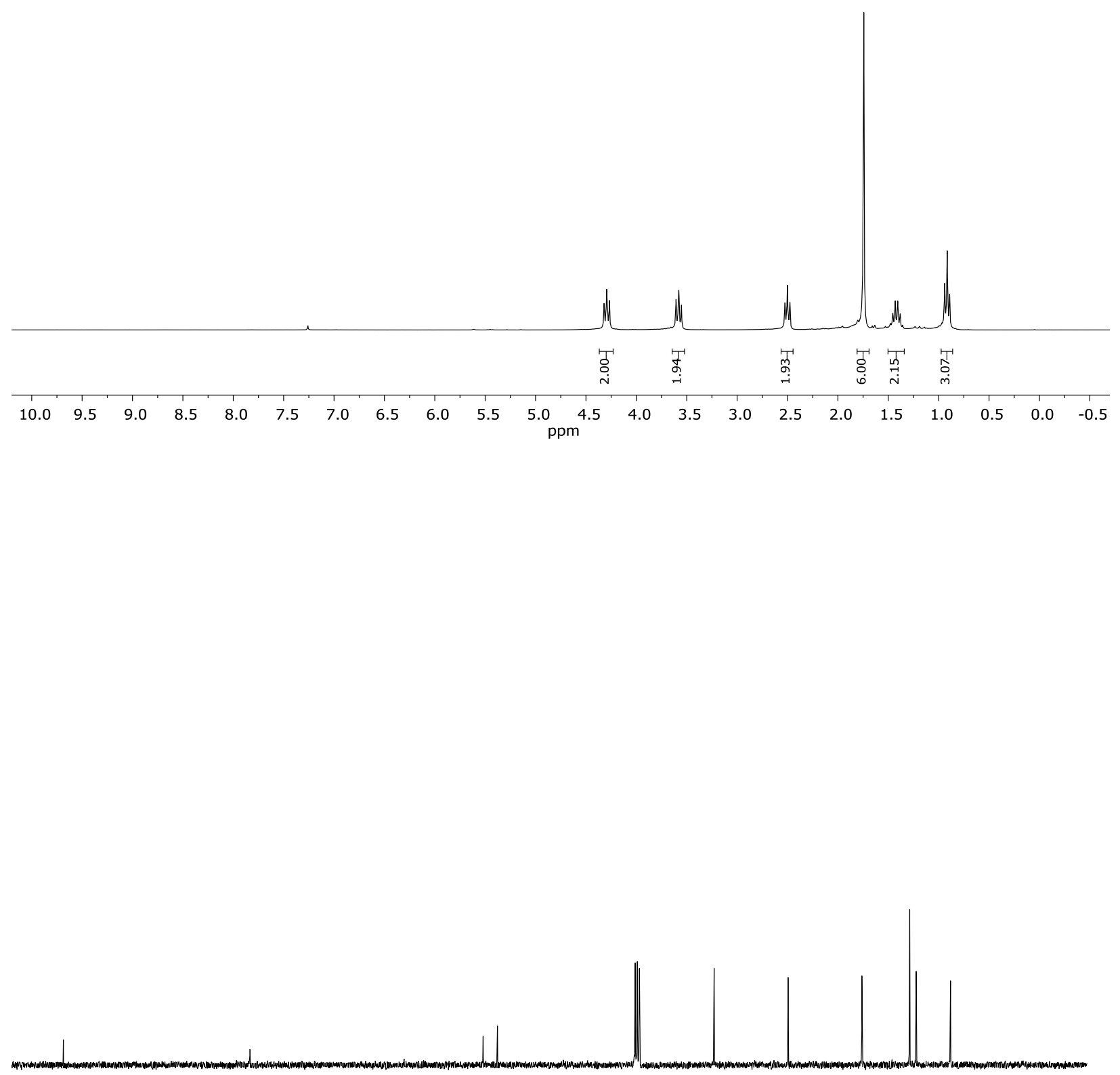

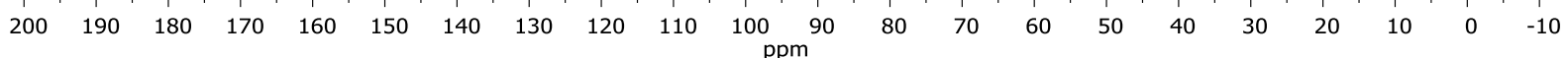




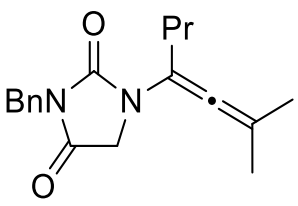

$1 n$
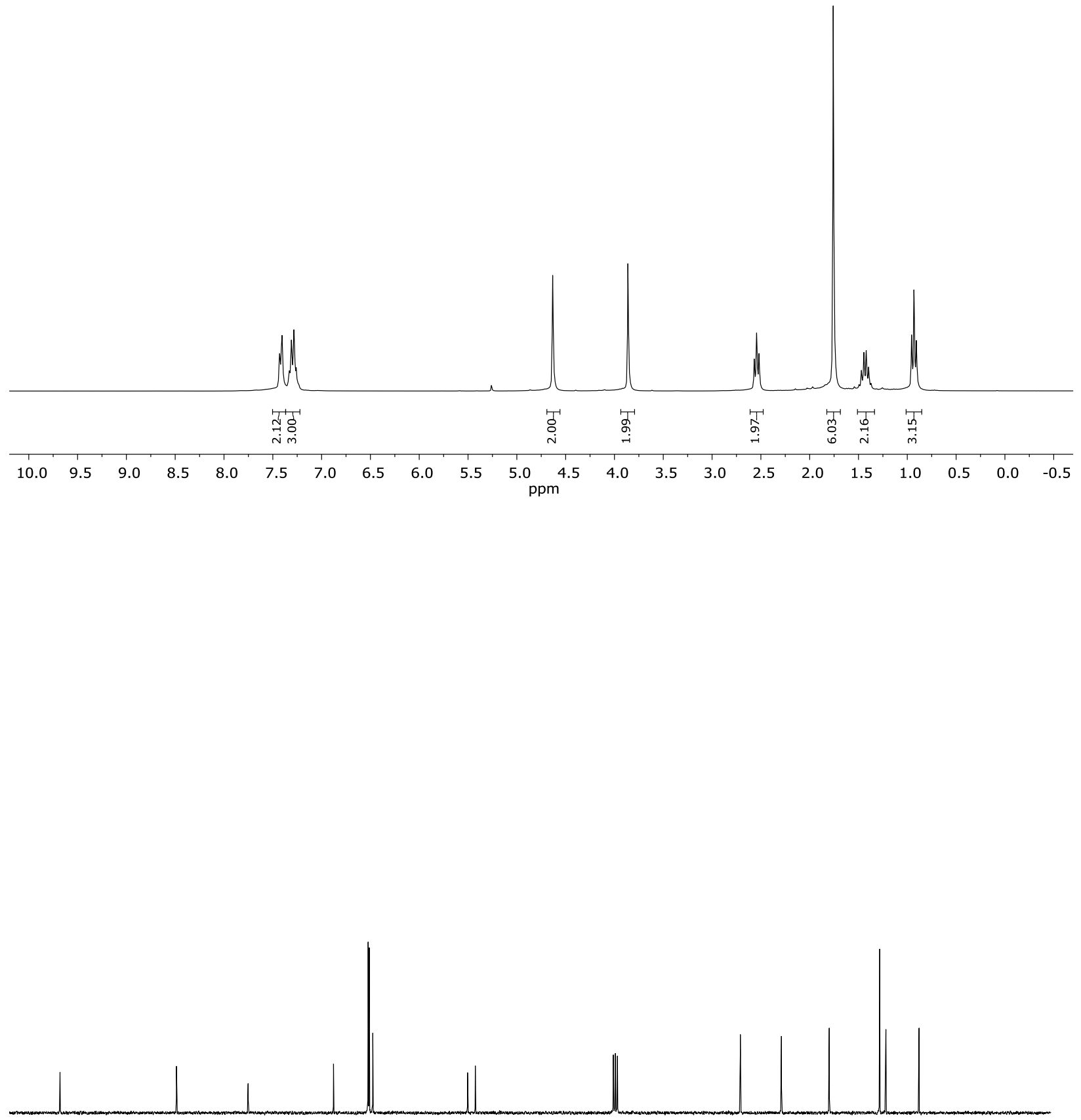

$\begin{array}{lllllllllllllllllllllllllllll}200 & 190 & 180 & 170 & 160 & 150 & 140 & 130 & 120 & 110 & 100 & 90 & 80 & 70 & 60 & 50 & 40 & 30 & 20 & 10 & 0 & -10\end{array}$ 


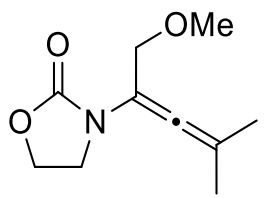

10
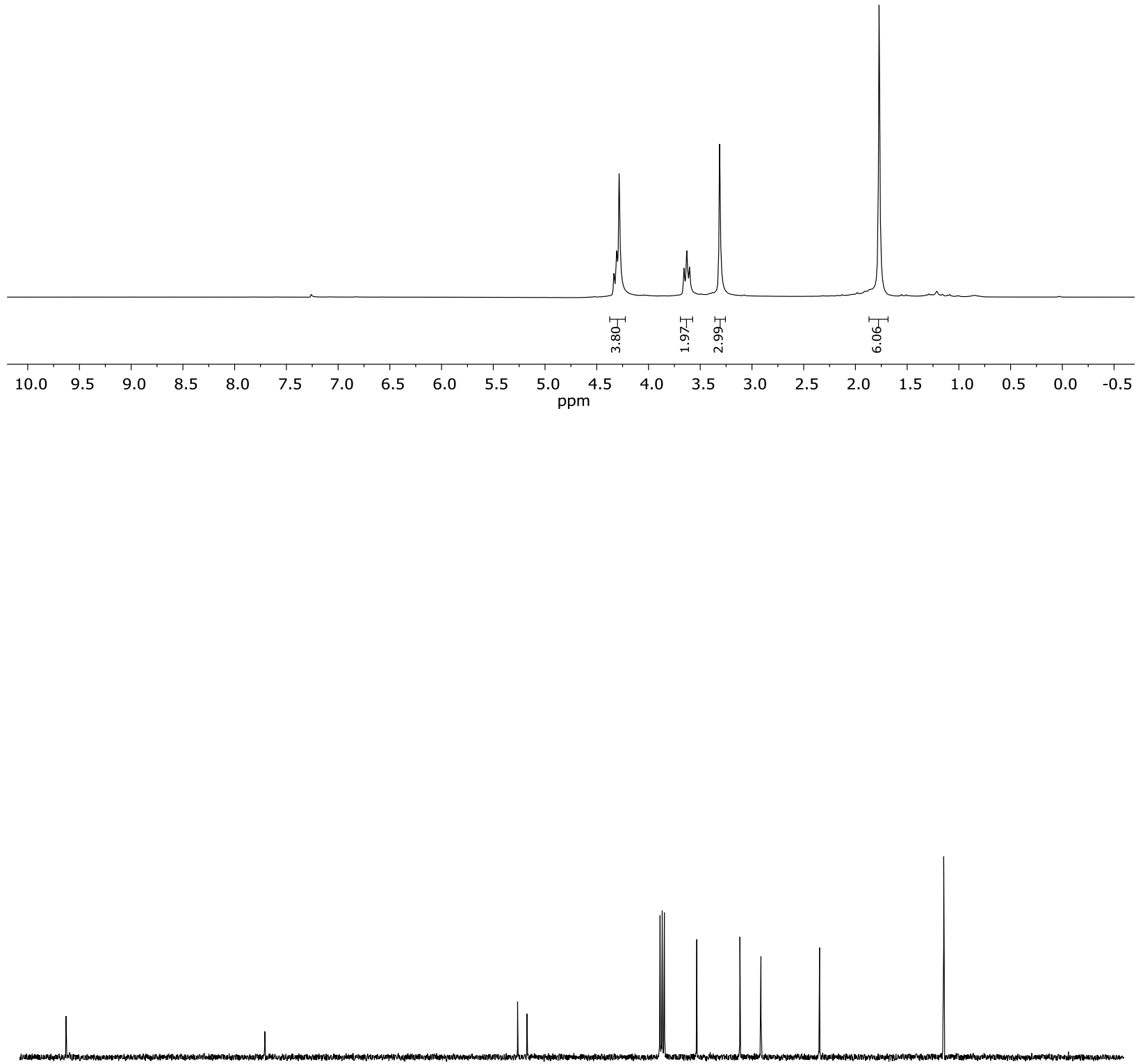

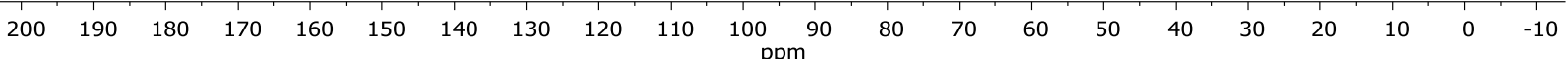




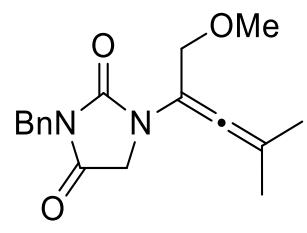

$1 p$
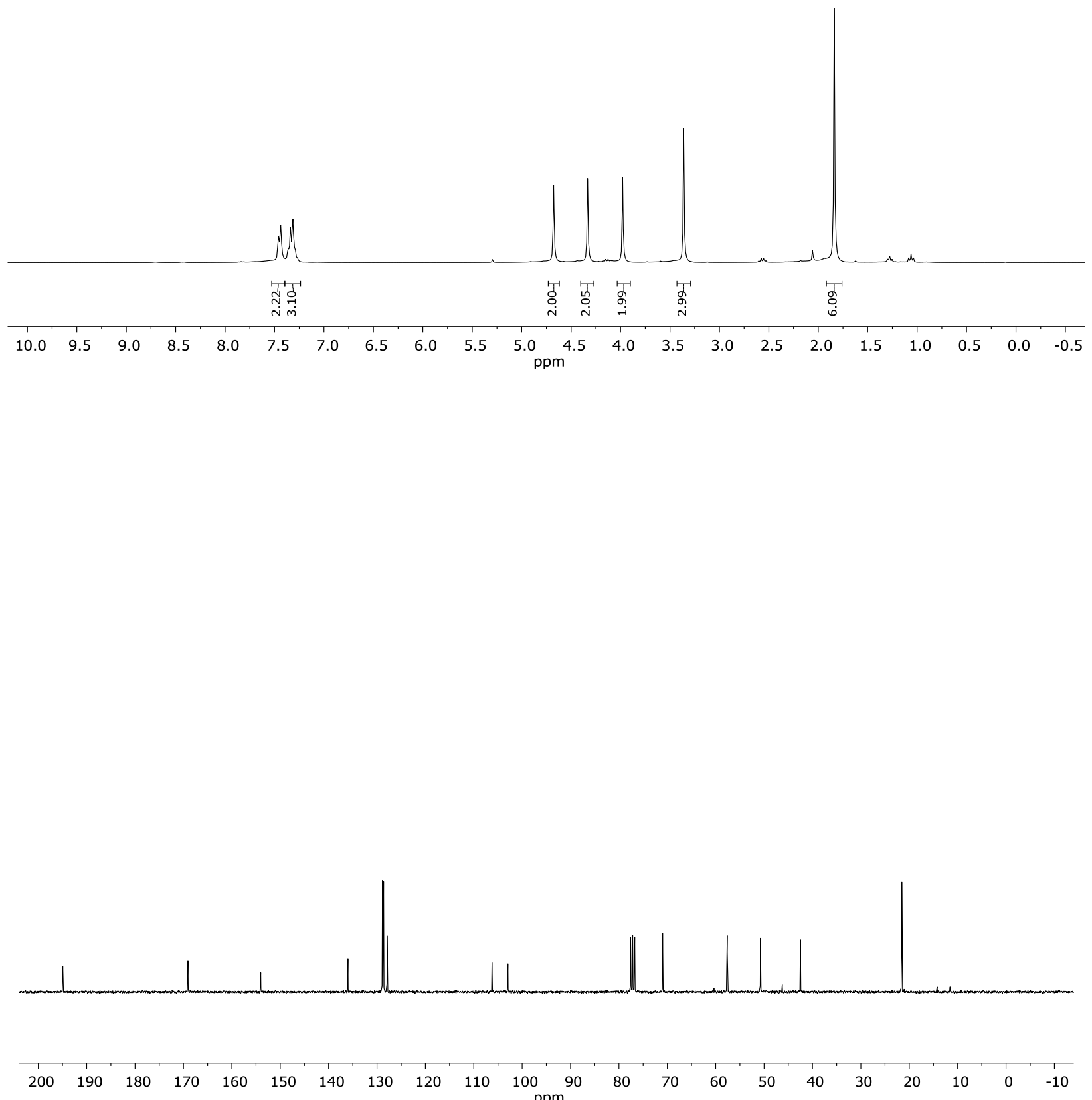


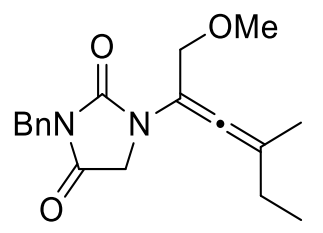

$1 q$
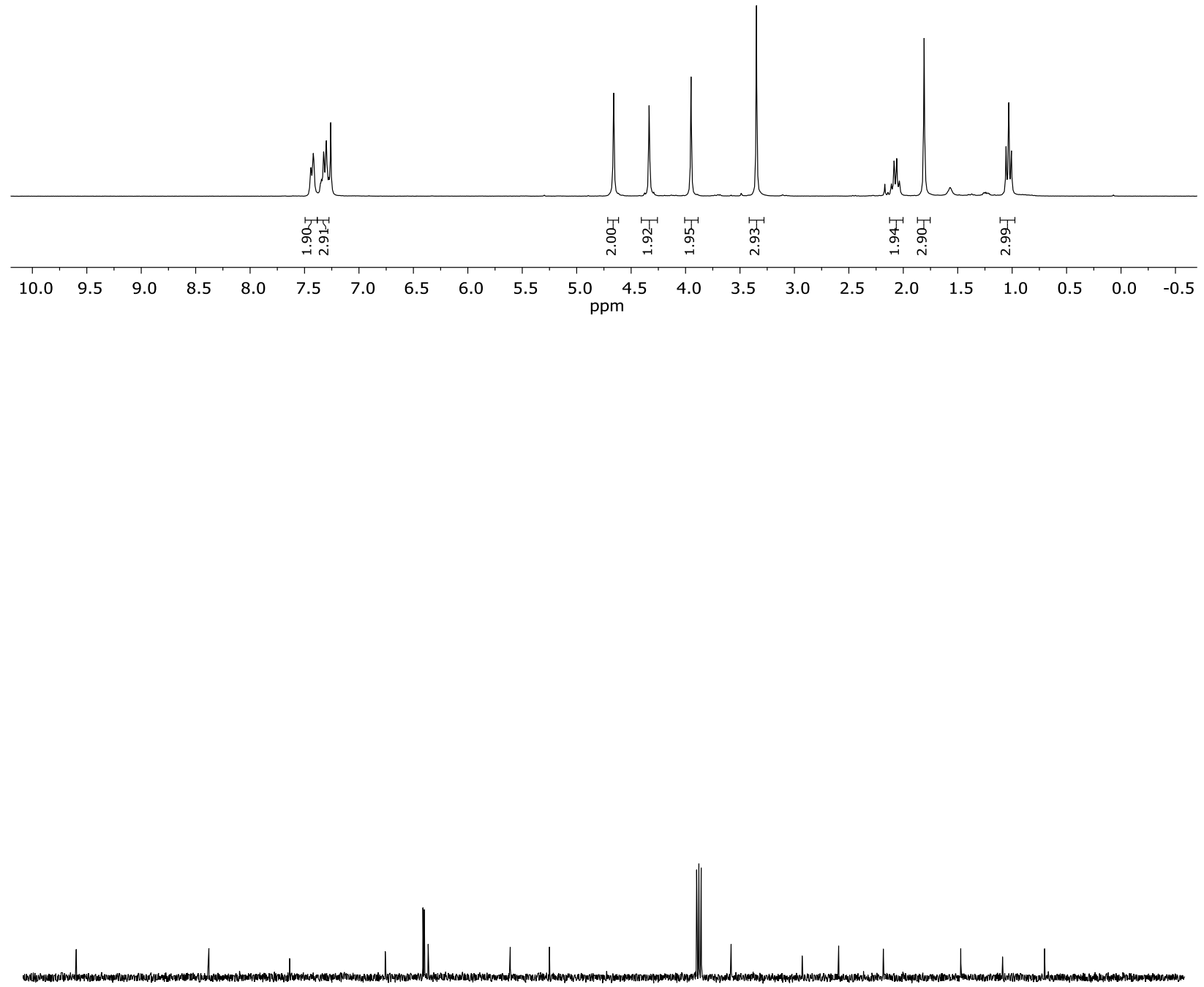

$\begin{array}{lllllllllllllllllllllll}200 & 190 & 180 & 170 & 160 & 150 & 140 & 130 & 120 & 110 & 100 & 90 & 80 & 70 & 60 & 50 & 40 & 30 & 20 & 10 & 0 & -10\end{array}$



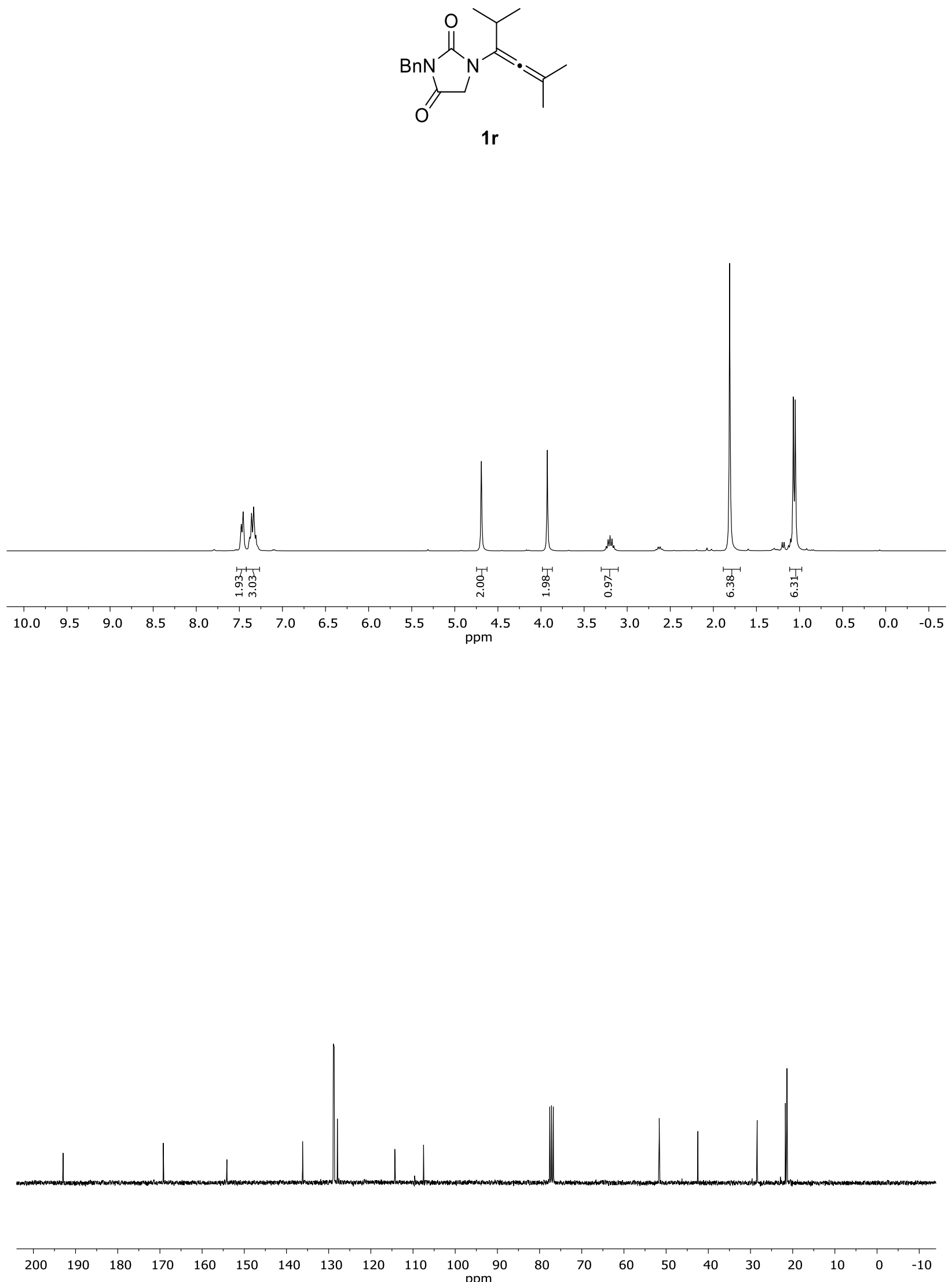


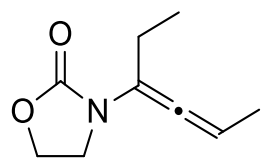

$1 \mathrm{~s}$
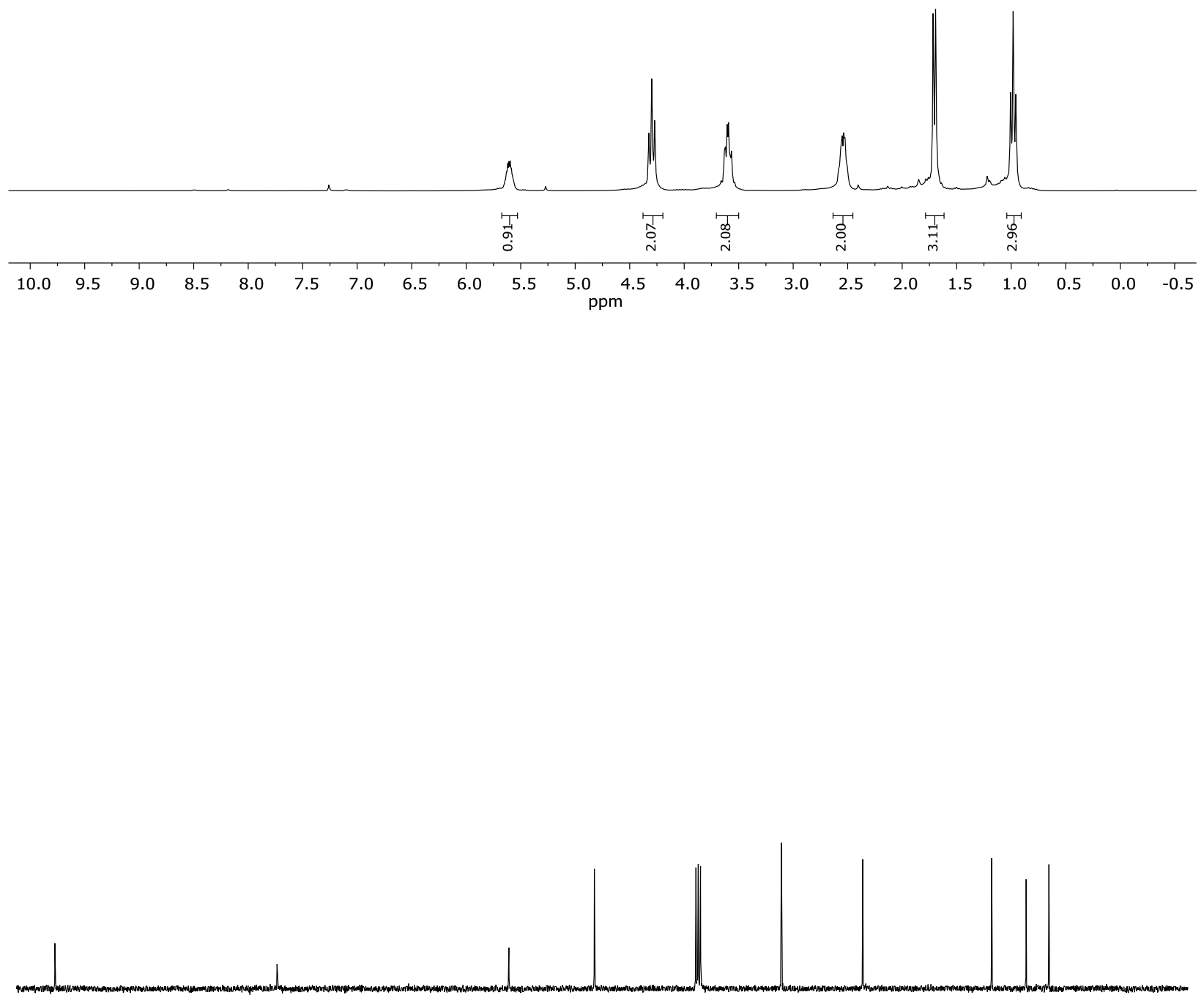

200

$190 \quad 180 \quad 170$

160150

140

100

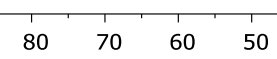




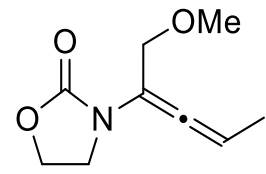

$1 \mathrm{t}$
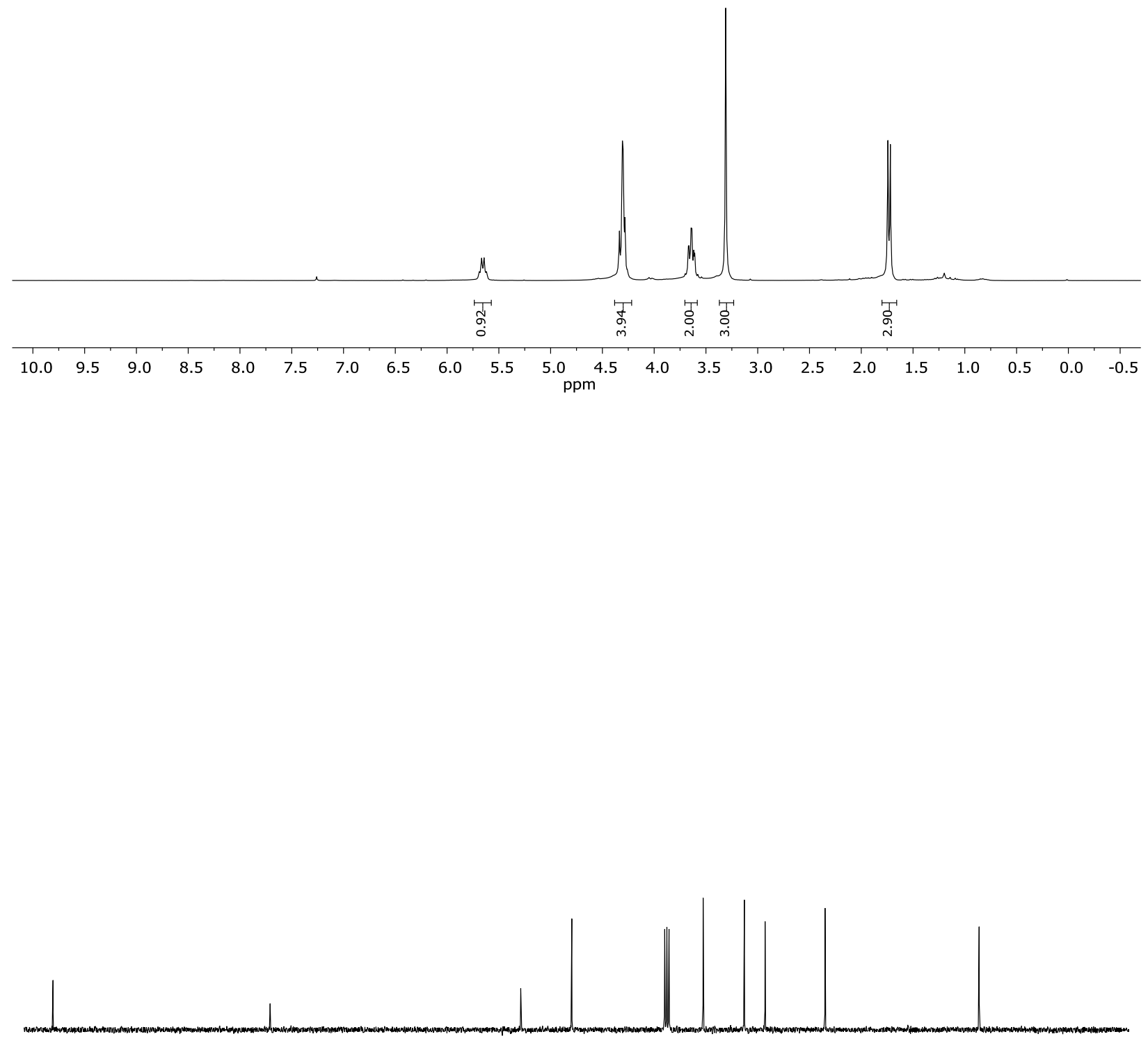

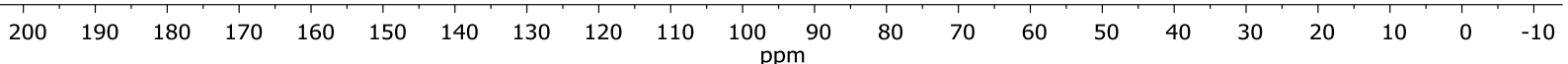



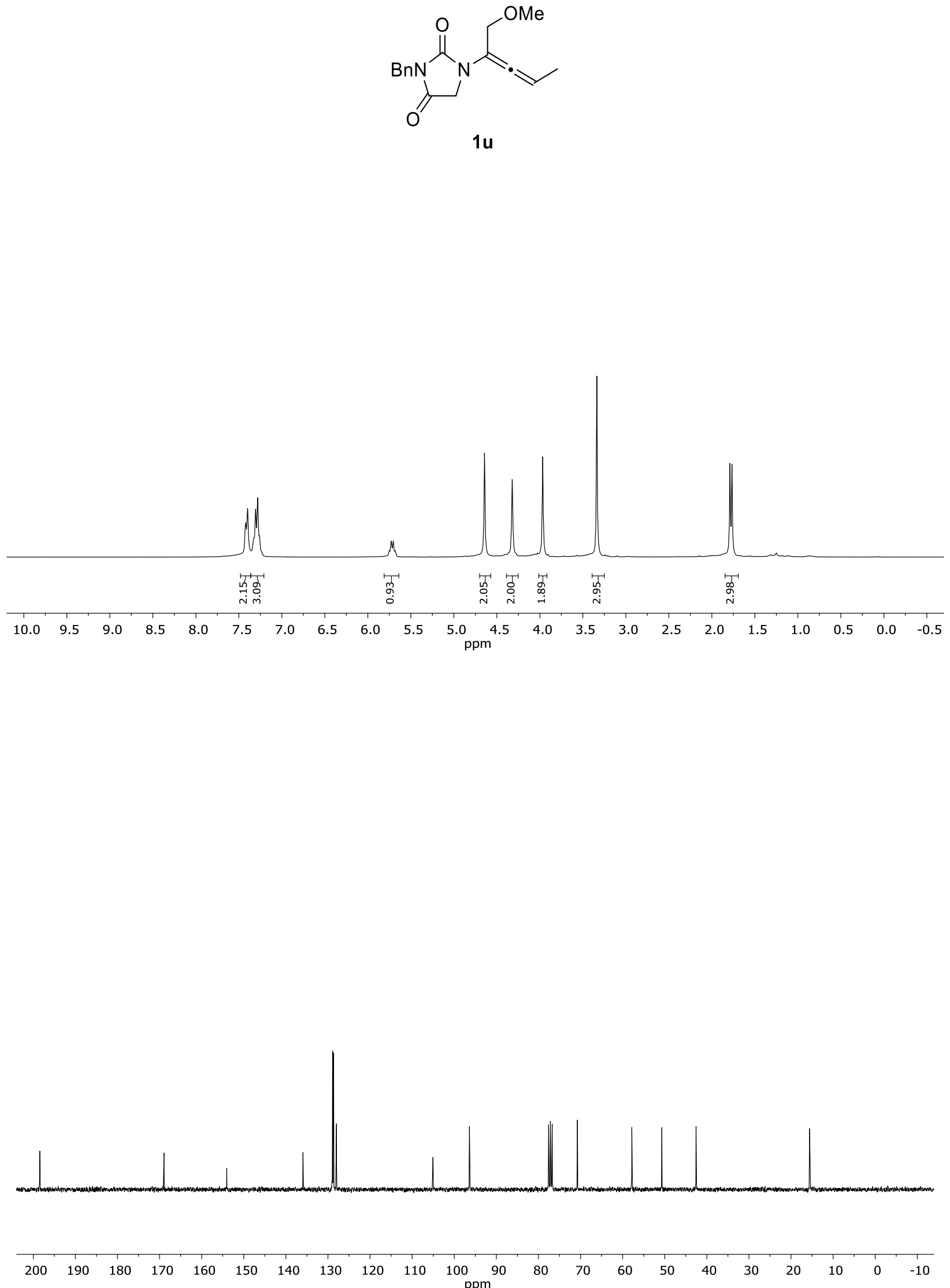

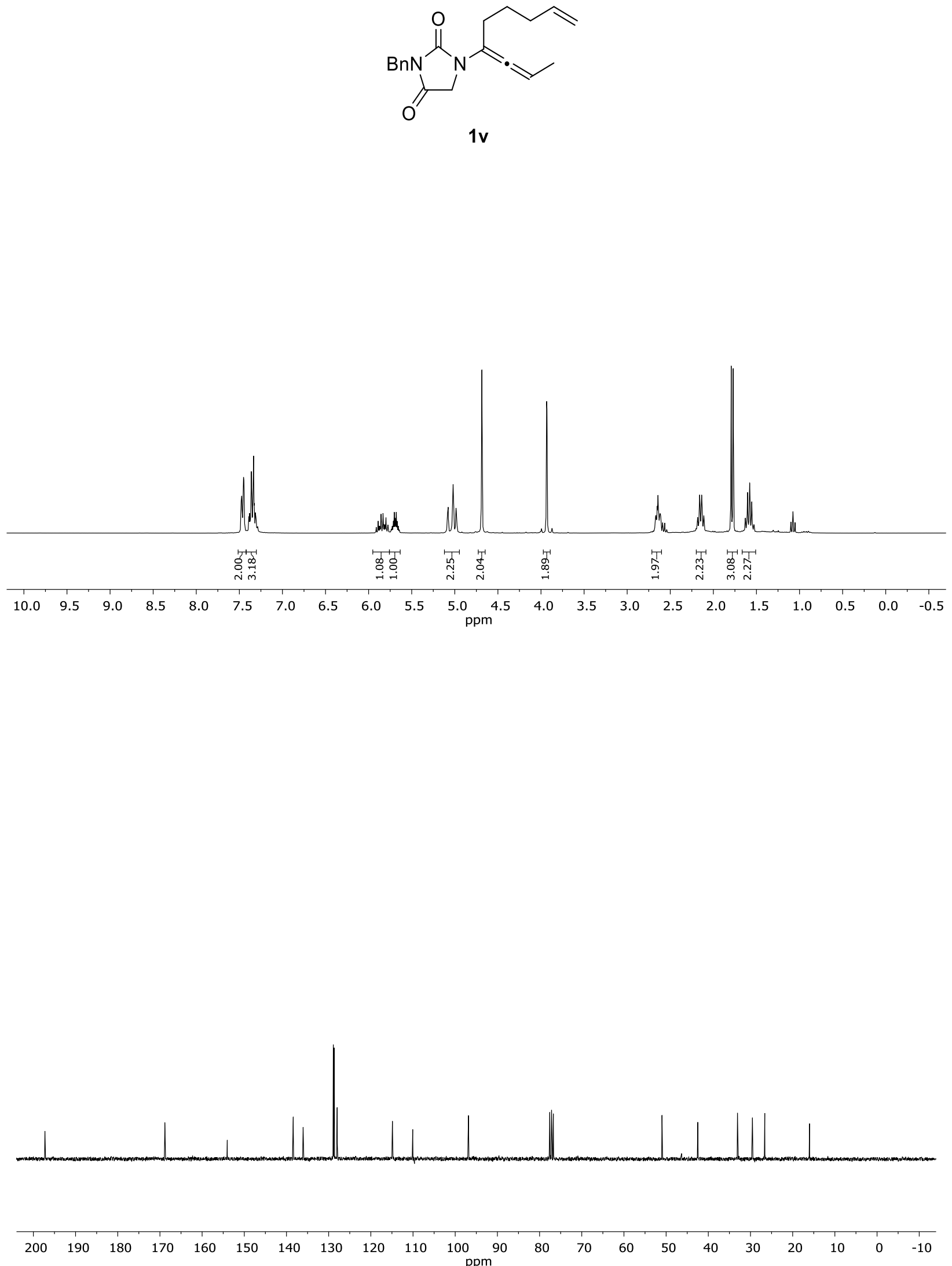


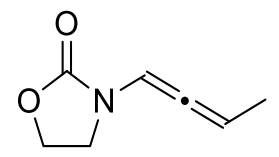

$1 w$
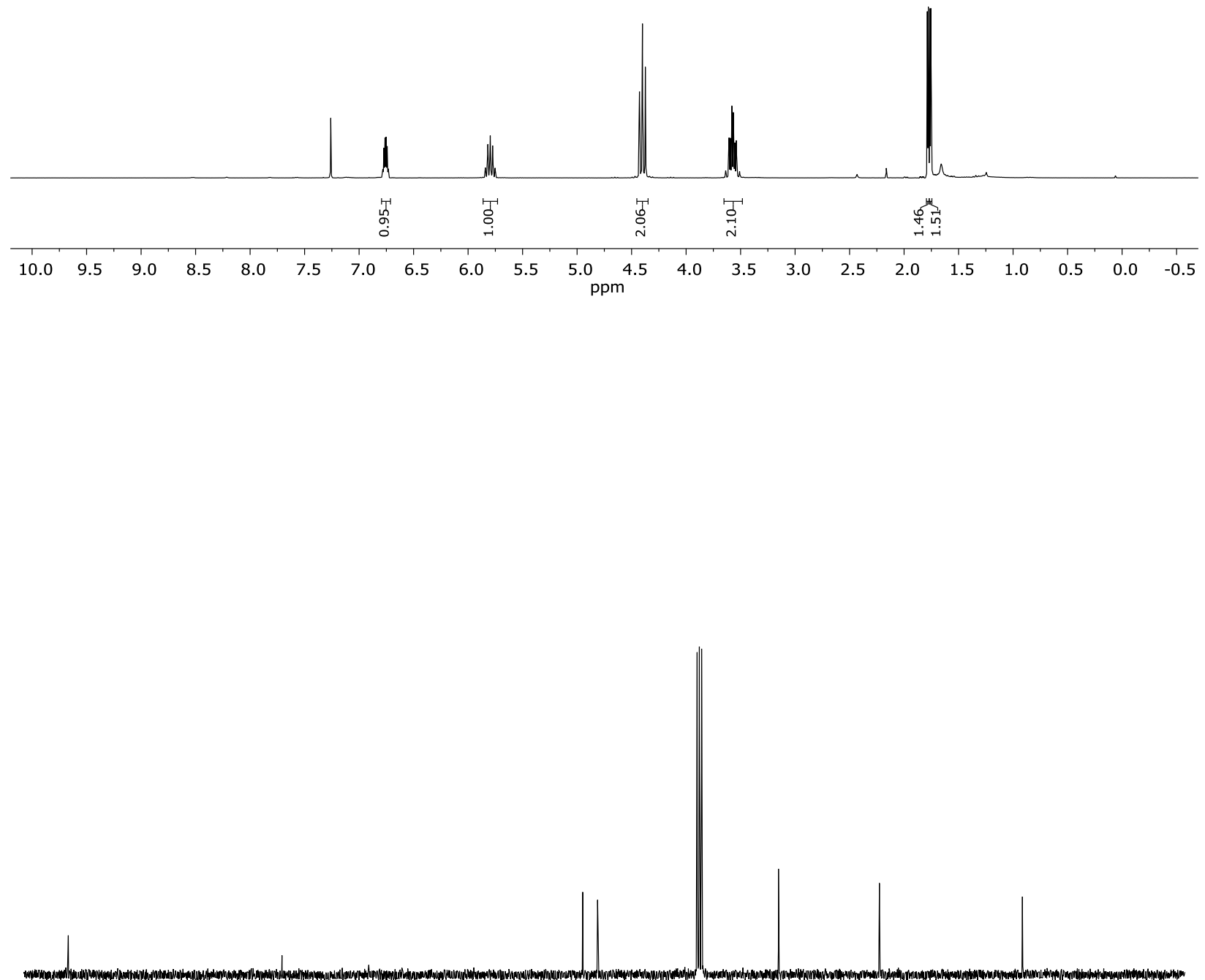

$\begin{array}{lllllllllllllllllllllll}200 & 190 & 180 & 170 & 160 & 150 & 140 & 130 & 120 & 110 & 100 & 90 & 80 & 70 & 60 & 50 & 40 & 30 & 20 & 10 & 0 & -10\end{array}$ 

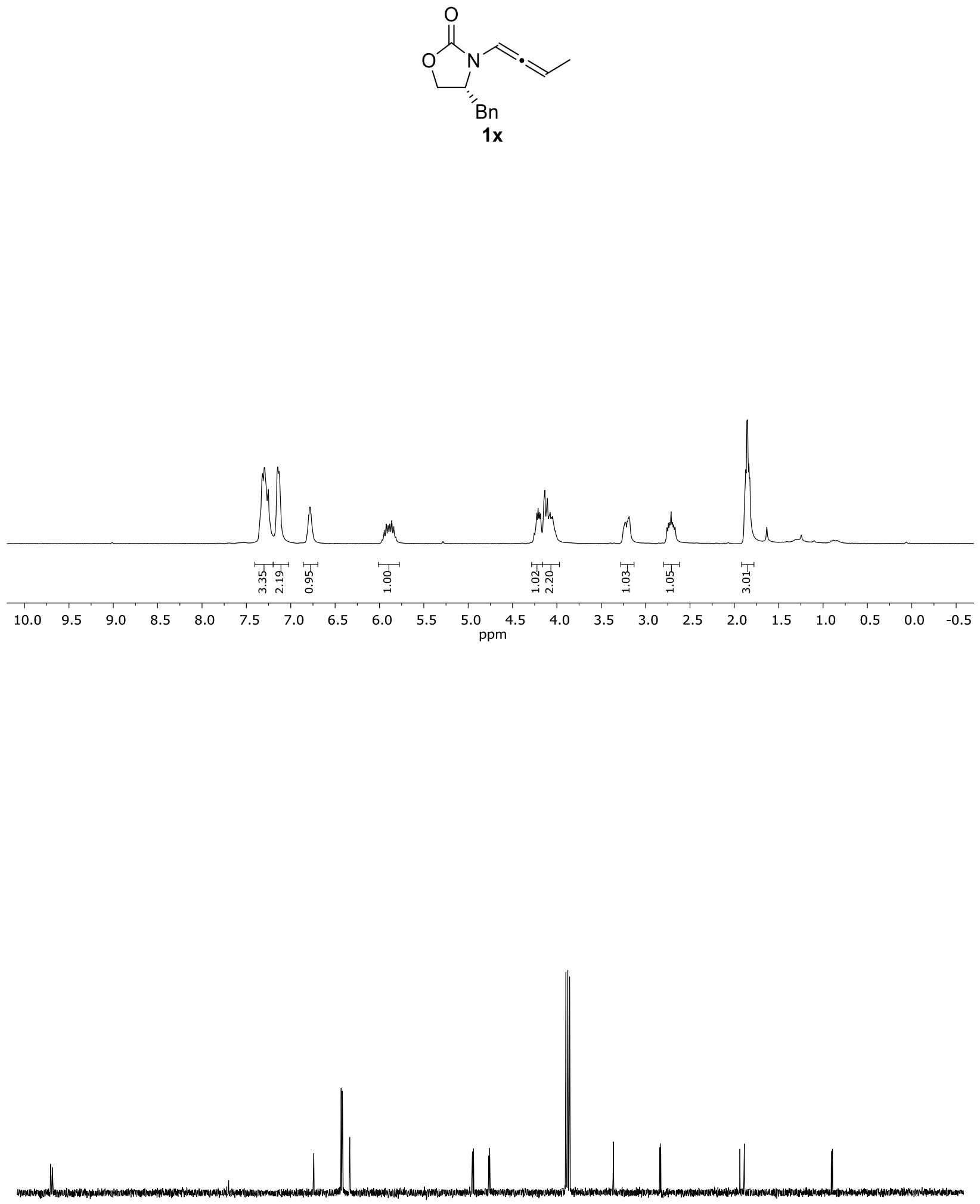

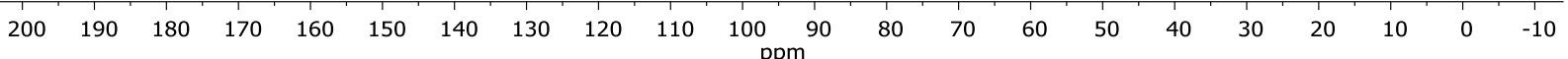



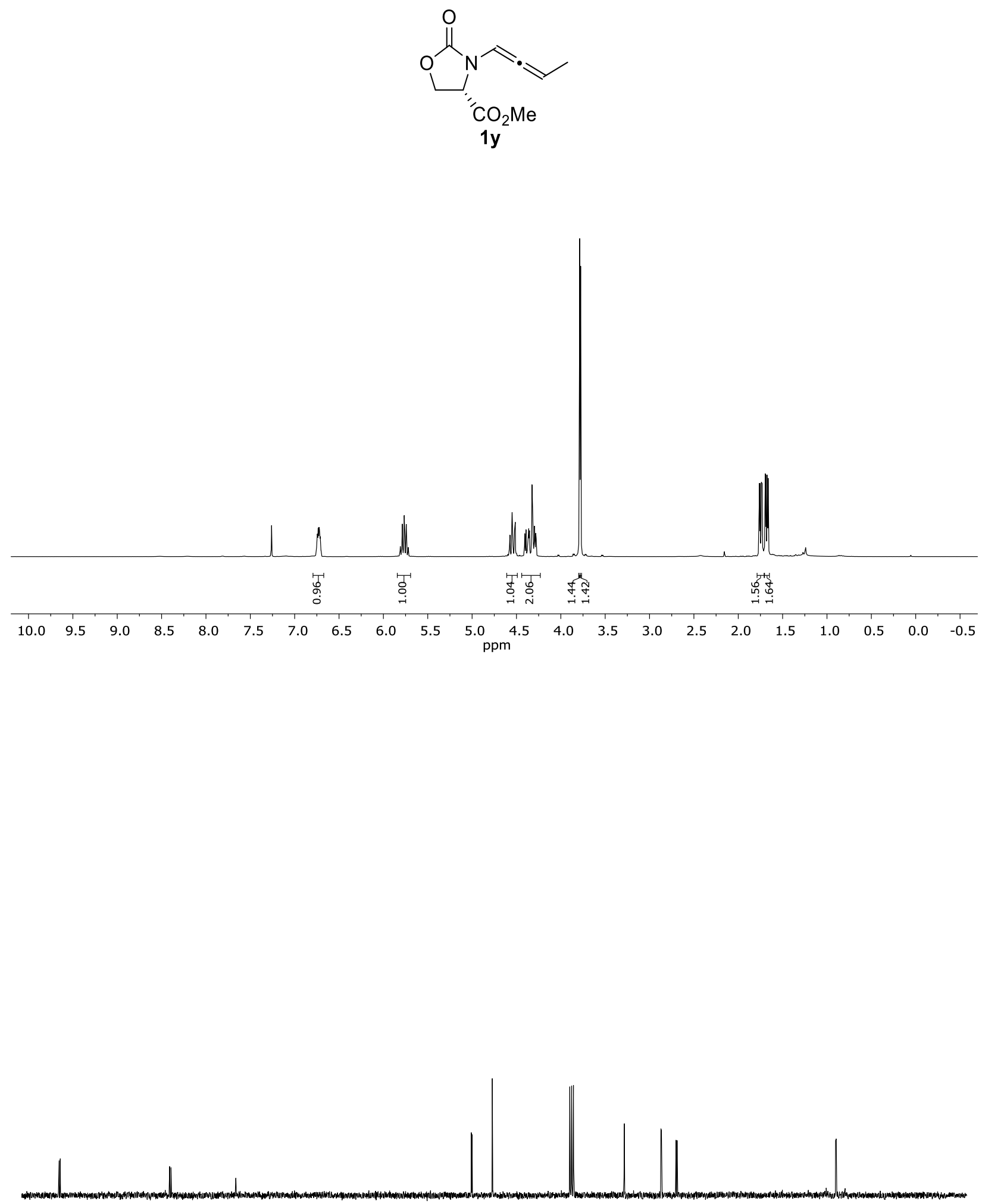

$\begin{array}{lllllllllllllllllllllll}200 & 190 & 180 & 170 & 160 & 150 & 140 & 130 & 120 & 110 & 100 & 90 & 80 & 70 & 60 & 50 & 40 & 30 & 20 & 10 & 0 & -10\end{array}$



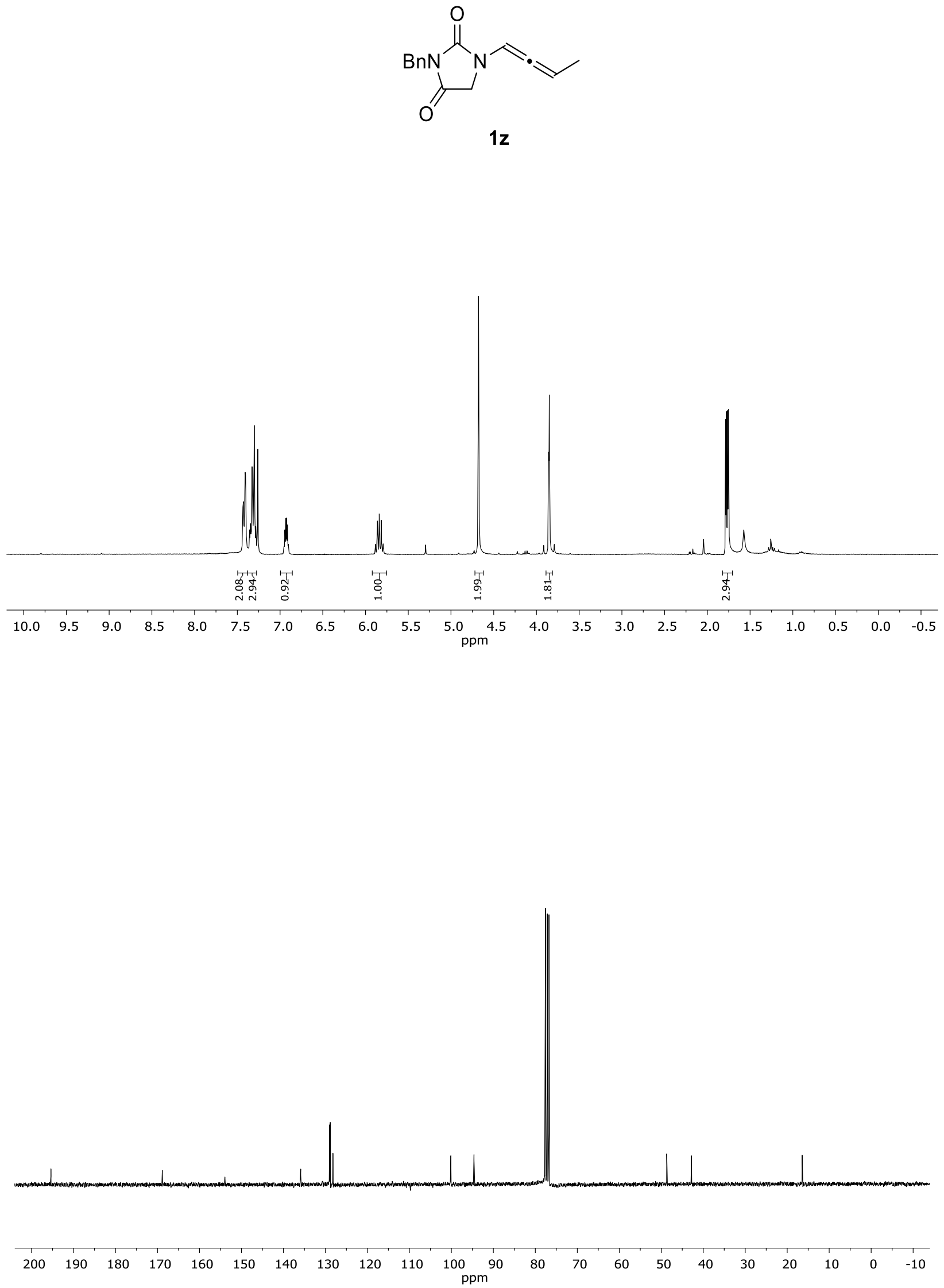